\title{
Locus-Specific DNA Methylation Editing in Mammalian Cells using a CRISPR-Based System
}

Jim Smith ${ }^{1}$, Rakesh Banerjee ${ }^{1}$, Reema Waly ${ }^{1}$, Arthur Urbano ${ }^{1}$, Gregory Gimenez ${ }^{1}$, Robert Day $^{2}$, Michael R. Eccles ${ }^{1}$, Robert J. Weeks ${ }^{1, *}$ and Aniruddha Chatterjee ${ }^{1, *}$

${ }^{1}$ Department of Pathology, Otago Medical School, University of Otago, Dunedin 9054, New Zealand

${ }^{2}$ Department of Biochemistry, Division of Health Sciences, University of Otago, Dunedin 9054, New Zealand

*Correspondence: aniruddha.chatterjee@otago.ac.nz (A.C); rob.weeks@otago.ac.nz (R.J.W)

Keywords: CRISPR; dCas9; DNA methylation; epigenetic editing; melanoma; mammalian cell lines

\begin{abstract}
DNA methylation is a key epigenetic modification implicated in the pathogenesis of numerous human diseases, including cancer development and metastasis. Gene promoter methylation changes are widely associated with transcriptional deregulation and disease progression. The advent of CRISPR-based technologies has provided a powerful toolkit for locus-specific manipulation of the epigenome. Here, we describe a comprehensive global workflow for the design and application of a dCas9-SunTag-based tool for editing a DNA methylation locus in human melanoma cells, alongside protocols for downstream techniques used to evaluate subsequent methylation and gene expression changes in methylation-edited cells. Using transient system delivery, we demonstrate both highly efficacious methylation and demethylation of the EBF3 promoter, a putative epigenetic driver of melanoma metastasis, achieving up to $304.00 \%$ gain of methylation and $99.99 \%$ relative demethylation, respectively. Further, we employ a novel, targeted screening approach to confirm minimal off-target activity and high on-target specificity of our editing sys-tem within our target locus.
\end{abstract}




\section{Introduction}

DNA methylation (5-methylcytosine; $5 \mathrm{mC})$ is a stable, and perhaps the most widely studied, epigenetic modification involved in the regulation of gene transcription $[1,2]$. Dysregulation of DNA methylation is implicated in the pathogenesis of numerous diseases. Aberrant DNA methylation in promoter regions of tumor-suppressor genes and global loss of DNA methylation has been strongly associated with the development and progression of many different tumors [3-5]. Classically, promoter DNA methylation is associated with transcriptional silencing [6]. However, several instances of promoter hypermethylationinduced gene activation have now been recorded [7-12]. We identified the $E B F 3$ gene as a putative epigenetic driver of melanoma metastasis [13] and in several other solid cancers [14], which shows paradoxical activation of transcription from highly methylated promoter. Understanding the precise mechanism of gene regulation via DNA methylation has great potential for advancing our understanding of disease pathophysiology and in identifying new targets for novel treatments [15]. Until now, it has been very difficult to establish true causality between DNA methylation changes and subsequent alterations in gene expression. However, with the advent of cutting-edge editing tools such as CRISPR, it is now possible to investigate the sequelae of aberrant methylation in diseases such as cancer in a causal manner $[16,17]$.

We have streamlined a method using the clustered regularly interspaced short palindromic repeats (CRISPR)-dCas9 system to facilitate site-specific editing of DNA methylation in mammalian cells $[18,19]$. Although we have used this system to methylate and demethylate the promoter region of $E B F 3$, using the CRISPR toolkit, the system described can be easily modified to target any known locus of interest within the genome, providing a highly selective method of epigenomic manipulation with minimal off-target impacts across the wider DNA methylome.

Our CRISPR-methylation system is based on an earlier system described by Huang et al (2017) [19], adapted for successful transient delivery into human melanoma cell lines and expanded 
to allow for targeted DNA demethylation alongside methylation. Immortalised cell lines are widely used as an experimental model for the fundamental investigation of tumour cell biology. DNA methylation status has been demonstrated to be well conserved between tumour tissue samples and derivative cell lines; therefore, cell lines provide an effective in vitro model for studying epigenetic alterations in cancer cells [4, 20, 21]. Our editing system comprises three main components: a dCas9-SunTag targeting protein; locus-specific guide RNA (gRNA; sgRNA) construct; and an effector construct for manipulating DNA methylation (Figure 1). The dCas9-SunTag construct is composed of a catalytically inactive Streptococcus pyogenes (S. pyogenes) Cas9 (dCas9) protein, fused to the SunTag (SUperNova TAGging) protein scaffold. dCas9 allows for RNA-programmable binding of our CRISPR-methylation editing system to a single target locus, without inducing cleavage of the underlying DNA sequence. Further, SunTag provides a repeating, epitope-based scaffold which is capable of binding multiple copies of our effector construct via short-chain variable fragment (scFv) domains [16]. dCas9-SunTag binding to a target genomic locus is directed by a unique gRNA construct. The S. pyogenes Cas9 module recognizes a 20 nt spacer sequence homologous to the target locus, which must immediately precede a 5' -NGG-3' protospacer adjacent motif (PAM) [22]. Once targeted binding of dCas9-SunTag to our locus of interest has occurred, up to ten effector constructs bind to the SunTag scaffold via scFv binding domains. Here, effector constructs refer to proteins with the capacity to induce active methylation or demethylation of $\mathrm{CpG}$ dinucleotides, including the catalytic domains of the human DNMT3A methyltransferase or TET1 dioxygenase, respectively. The catalytic domain of the TET1 protein is preferred over the full length construct due to the difficulties with transfecting very large modules [23]. Collectively, these three constructs form our CRISPR-methylation editing system, with the capacity to induce active changes in DNA methylation at specific genomic loci. Here, broadly applicable protocols are detailed for gRNA design, and delivery of our CRISPR-methylation editing system into human melanoma cell lines.

\section{Materials and Methods}


A full list of reagents and equipment for this protocol are detailed in Appendix A.

\section{1 gRNA Design for CRISPR-Methylation Editing}

The simple design of gRNA sequences for CRISPR experiments using the cloud based Benchling platform (http://benchling.com) has been previously described [24] and can be applied to any known target sequence. Benchling and other CRISPR gRNA design tools use an algorithm-based approach to generate potential gRNA sequences specific to a target locus with respect to PAM site requirements. In this protocol, we use the S. pyogenes Cas9 system, which will limit target gRNA sequences to those immediately preceding 5'-NGG-3' [22]. Each algorithm then weights prospective gRNA sequences based on their projected on-target specificity and off-target activity. In CRISPR-methylation editing, minimising off-target activity is crucial to establishing causal roles for DNA methylation in pathways such as transcriptional regulation (see Section 2.5 for further discussion of off-target effects and an effective protocol for the targeted evaluation of off-target activity). When selecting gRNA sequences for methylation-editing, there are several additional factors that need to be considered. Firstly, as methylation-editing uses a dCas9-SunTag component, binding of dCas9 to the target locus will physically obstruct $20-30$ bp directly overlying the gRNA target sequence [25]. The limited evidence currently available suggests that dCas9-SunTag-based systems are able to achieve efficient changes in DNA methylation up to around $1 \mathrm{~kb}$ from the PAM site [18]. Following design and gRNA selection, order oligonucleotides which represent both strands of the gRNA sequence; each oligonucleotide pair should have the following sequences:

Forward oligonucleotide: $\quad$ 5'-CACCG(N)20-3'

Reverse oligonucleotide: $\quad 3$ '-C(N')20CAAA-5'

(N)20 denotes the unique user-designed guide sequence in the forward oligonucleotide and (N')20 the reverse complement of the guide sequence in the reverse oligonucleotide. Each 
gRNA sequence requires, if not already present, the addition of a 5' guanine residue (bold) which serves as a transcriptional initiation site for the U6 promoter in the final gRNA construct (a corresponding cytosine residue is therefore added to the reverse oligonucleotide sequence). A 5'-CACC sequence and 3'-CAAA sequence are added to the forward and reverse oligonucleotide, respectively, to generate complementary 'overhanging' sequences for 'inframe' cloning into the BsmBI-digested pLKO5.sgRNA.EFS.tRFP657 vector. If possible, oligonucleotides should be ordered with pre-phosphorylated 5' ends, removing the requirement for phosphorylation during gRNA construct preparation.

\subsection{CRISPR-Methylation Plasmid Preparation}

\subsubsection{Preparation of dCas9-SunTag and Effector Constructs}

The dCas9-SunTag plasmid construct used here was obtained from Addgene (Catalogue \#60903; pHRdSV40-dCas9-10xGCN4_v4-P2A-BFP). Construct preparation for the gRNA and effector proteins was based on the previously described protocols of Huang et al (2017) [13], using plasmids also available from Addgene. As per these vector construction protocols, pLKO5.sgRNA.EFS.tRFP657 (Catalogue \#57824) was used as a vector for all unique gRNA constructs and pHRdSV40-scFv-GCN4-sfGFP-VP64-GB1-NLS (Catalogue \#60904) was used as a vector for all effector proteins. All gRNA and effector constructs were prepared via restriction cloning, as per the cited protocols [19]. In brief, pHRdSV40-scFv-GCN4-sfGFPVP64-GB1-NLS plasmids were restriction digested using RsrII and SpeI endonucleases overnight at $37^{\circ} \mathrm{C}$. Effector protein sequences were amplified from respective parent plasmids using primers with added RsrII and SpeI recognition sites and, subsequently, ligated into the digested vector. For gRNA construct preparation, pLKO5.sgRNA.EFS.tRFP657 plasmids were digested using BsmBI overnight at $55^{\circ} \mathrm{C}$ then the ends dephosphorylated using rSAP (shrimp alkaline phosphatase). Respective forward and reverse gRNA oligonucleotides were then annealed and cloned into the digested vector (see Section 2.2.2). 
For our work, all unique gRNA sequences were designed and selected as per the protocol detailed in the procedure section (see 2.1 gRNA Design for CRISPR-Methylation Editing). Two independent effector constructs were generated, containing sequences for the human DNMT3A protein and the catalytic domain of human TET1, respectively. The sequences for these effector constructs were derived from the following commercially available plasmids, respectively: Fuw-dCas9-DNMT3A (Catalogue \#84476) and Fuw-dCas9-TET1CD (Catalogue \#84475) [26]. It should be noted that if different plasmids to those stated are used for methylation-editing experiments, fluorophore selection within the plasmids is a key consideration to facilitate effective FACS (i.e., if using different plasmids, ensure any tagged fluorophores have sufficiently different emission spectra to allow for effective sorting of triplepositive transfected cells).

With respect to optional assessment of gRNA on-target editing specificity and potential offtarget activity (see 2.6 gRNA Evaluation for On-Target Specificity and Off-Target Activity), our rapid screening protocol uses the active Cas9 construct pSpCas9(BB)-2A-GFP (Catalogue \#48138) [27], also available from Addgene. This construct is propagated and isolated in the same manner as for the other plasmids used in this protocol. Simple screening of the isolated pSpCas9(BB)-2A-GFP plasmid DNA to confirm that the construct is of the correct size can be performed using EcoR1 restriction digest, which will generate two fragments of 8,505 bp and 783 bp, respectively.

\subsubsection{Preparation of Guide RNA (gRNA) Constructs}

Begin gRNA construct preparation by performing restriction digestion of $25.0 \mu \mathrm{L}$ (up to 10 $\mu \mathrm{g}$ ) of the pLKO5.sgRNA.EFS.tRFP657 vector using $2.0 \mu \mathrm{L}$ (20 units) of BsmBI restriction endonuclease overnight at $55{ }^{\circ} \mathrm{C}$, in a total reaction volume of $105.0 \mu \mathrm{L}$, made up with appropriate enzyme buffer and water. Add $2.0 \mu \mathrm{L}$ ( 2 units) of shrimp alkaline phosphatase to the digested product and incubate at $37{ }^{\circ} \mathrm{C}$ for $30 \mathrm{~min}$, followed by $65{ }^{\circ} \mathrm{C}$ for $5 \mathrm{~min}$ to dephosphorylate the free ends of the digested vector. Purify the dephosphorylated vector using the DNA Clean and Concentrator-5 Kit or equivalent. 
For annealing of each respective gRNA, combine $1.0 \mu \mathrm{L}$ of sense oligonucleotide $(100 \mu \mathrm{M})$, $1.0 \mu \mathrm{L}$ of antisense oligonucleotide $(100 \mu \mathrm{M}), 1.0 \mu \mathrm{L}$ of T4 DNA Ligase Buffer and $7.0 \mu \mathrm{L}$ of water. Mix by pipetting and perform thermal cycling with the following protocol: $95{ }^{\circ} \mathrm{C}$ for 2:30 minutes; $-1.0^{\circ} \mathrm{C}$ per cycle for 10 seconds (repeat x 72 ); infinite hold at $22{ }^{\circ} \mathrm{C}$. Dilute the annealed gRNA sample 1:500, then ligate into the digested pLKO5.sgRNA.EFS.tRFP657 vector at room temperature overnight using T4 DNA Ligase. Transform the ligated construct into competent $E$. coli cells for propagation on LB agar containing $100 \mu \mathrm{g} / \mathrm{mL}$ ampicillin, overnight at $37^{\circ} \mathrm{C}$. Confirmation of the inserted gRNA sequence can be performed via Sanger sequencing of single colonies using the U6 promoter primer (5'TTTGCTGTACTTTCTATAGTG-3'), prior to bulk culture and transfection.

\subsection{Transient Delivery of the CRISPR-Methylation Editing System}

We recommend creating a transfection plan prior to each transfection experiment in order to establish reagent requirements and streamline the transfection process. In particular, detailed planning is important when performing complex transfections involving multiple constructs (i.e., when multiplexing gRNA molecules or using different effector constructs). This protocol describes a general method for the transient delivery of our CRISPR-methylation editing system into human melanoma cell lines via lipofection. It should be noted that other transfection methods may be better suited to different cell lines. Lipofection is performed using the Lipofectamine 3000 transfection system, with slight variation from the manufacturer's protocol. Cells positive for successful plasmid delivery are subsequently sorted by FACS at 72-hours post-transfection.

\subsubsection{Cell Culture}

For our optimized protocol, human melanoma cell lines WM115, CM150-Post and NZM40 were used. Cell line WM115 was obtained from America Type Culture Collection (Manassas, VA) (ATCC® CRL-1675TM). WM115 was cultured in Minimum Essential Media-Alpha 
(MEM- $\alpha$ ) (Invitrogen) supplemented with 1\% penicillin-streptomycin (Gibco, NY, USA) and $10 \%$ foetal calf serum (FCS). CM150-Post is a cell line established from patients entered into the Roche "BRIM II" phase II study of vemurafenib in patients who had previously failed treatment [28]. CM150-Post was cultured in Dulbecco's Modified Eagle Medium (DMEM) (Invitrogen) supplemented with 10\% FCS and 1\% penicillin-streptomycin as previously described [3]. NZM40 was generously provided by Professor Baguley (University of Auckland). NZM40 was cultured in MEM- $\alpha$ supplemented with 5\% FCS, $1 \%$ penicillinstreptomycin and $0.1 \%$ insulin-transferrin-selenium (Roche). All cell lines were cultured under standard conditions $\left(5 \% \mathrm{CO} 2,21 \% \mathrm{O} 2,37^{\circ} \mathrm{C}\right.$, humidified atmosphere $)$. Low passage and healthy conditions are essential to ensuring optimal transfection results. Cell lines were defrosted approximately one week prior to transfection, grown until $>85 \%$ confluent in a 75 $\mathrm{cm}^{2}$ cell culture flask, then passaged to a $175 \mathrm{~cm}^{2}$ cell culture flask.

Melanoma cells were propagated in appropriate culture medium until $>85 \%$ confluent. Appropriate culture medium and the length of time for cells to reach confluency will depend on the individual cell line. For best results, the following steps should be performed whilst the DNA-lipid complex(es) is/are incubating (see 2.3.3 Transfection). Trypsinise cells and transfer to an appropriate tube, then centrifuge for $5 \mathrm{~min}$ at $300 \mathrm{rcf}$. Remove culture medium and resuspend cells in an appropriate volume of culture medium for counting. Count cells and resuspend in culture medium to $5.0 \times 10^{5} / \mathrm{mL}$.

\subsubsection{DNA Preparation}

Propagate each construct in E. coli cells overnight, with appropriate antibiotic selection. For our work, we use $200-500 \mathrm{~mL}$ of each respective culture in LB broth plus $100 \mu \mathrm{g} / \mathrm{mL}$ ampicillin, cultured overnight at $37^{\circ} \mathrm{C}$, shaken at $200 \mathrm{rpm}$. Isolate plasmid DNA for each respective construct using an appropriate method. Isolating high-quality plasmid DNA with minimal bacterial endotoxin contamination is crucial for maximizing transfection efficiency and cell viability. Our preferred method for plasmid isolation is using the GenCatch ${ }^{\mathrm{TM}}$ Plasmid 
Plus DNA Maxiprep Kit. For convenience, these steps can be performed in advance and plasmid DNA can be stored at $4{ }^{\circ} \mathrm{C}$ until required for transfection. We recommend performing these steps up to several days in advance to minimise workload on the day of transfection. To confirm the identity of isolated plasmid DNA samples, Sanger sequencing or restriction digest may be used. For a simple restriction digest screen, it is possible to use the restriction enzymes which were used for cloning in reagent setup (e.g., RsrII/SpeI for effector protein construct(s)).

Prepare DNA combinations for transfection in sterile $1.5 \mathrm{~mL}$ microcentrifuge tubes by combining each respective construct in a 1:1:1 ratio, to the amount of $1500 \mathrm{ng}$ total DNA. Each DNA combination must include dCas9-SunTag, plus gRNA(s), plus an effector protein construct. One or more unique gRNA molecules may be used (i.e., for targeting multiple genomic loci). If using multiple gRNAs, the total amount of gRNA DNA should still equal the DNA of each other construct (i.e., $500 \mathrm{ng}$ total). For example, to perform targeted demethylation at locus $\mathrm{X}$, we would produce a plasmid DNA combination containing $500 \mathrm{ng}$ of our dCas9-SunTag, $500 \mathrm{ng}$ of gRNA designed to target locus X, and $500 \mathrm{ng}$ of our effector construct containing the TET1 catalytic domain. To target locus X and Y, we would instead use $250 \mathrm{ng}$ of gRNA for targeting locus X, plus $250 \mathrm{ng}$ of gRNA for targeting locus Y. For each run of transfections, include an empty tube which will be used for a negative control. Combinations can be prepared the day before transfection if desired. Mix combinations and spin briefly just prior to transfection.

\subsubsection{Transfection}

Each step of the transfection process should be performed in a sterile cell culture hood. It should be noted that we use a reverse transfection method, which deviates from the standard procedure recommended by the manufacturer (i.e., resuspended cells are added to each well containing transfection reagents). First, add $5 \mu \mathrm{L}$ P3000 reagent to $125 \mu \mathrm{L}$ Opti-MEM Serum-Free Medium, per transfection reaction to be performed. Suspend each pre-prepared DNA combination in $130 \mu \mathrm{L}$ of combined P3000 reagent and Opti-MEM Serum-Free Medium. Next, 
dilute $8 \mu \mathrm{L}$ of Lipofectamine 3000 reagent in $125 \mu \mathrm{L}$ of Opti-MEM Serum-Free Medium, per reaction, and add $133 \mu \mathrm{L}$ of combined Lipofectamine 3000 and Opti-MEM Serum-Free Medium to each suspended DNA combination. Incubate at room temperature for 15-20 min to allow for the formation of DNA-lipid complexes. For negative control samples, add all transfection reagents to an empty tube for transfection (i.e., as a negative control for FACS, cells will be exposed to all transfection reagents but no DNA, which provides a better control for FACS gating). Once each DNA-lipid combination has been incubated for 15-20 min, add each respective combination $(263 \mu \mathrm{L})$ to an individual well of a 6-well cell culture plate. Next, add $1 \mathrm{~mL}$ of suspended cells in culture medium $\left(5.0 \times 10^{5}\right)$ to each well. Add an additional 1$2 \mathrm{~mL}$ of culture medium to each well, bringing the total volume to 2-3 $\mathrm{mL}$ per well. Incubate under appropriate cell culture conditions.

\subsubsection{Cell Recovery and Maintenance}

After the optimal exposure period, remove transfection reagents from the cells. The optimal exposure period to transfection reagents needs to be determined for each respective cell line and is defined as the length of exposure time to transfection reagents resulting in the highest percentage transfection efficiency without a substantial negative impact on cell viability (as assessed during FACS at 72 hours post-transfection). For our work, optimal exposure periods were 6 hours (WM115, CM150-Post) and 12 hours (NZM40), respectively. Wash with $1 \mathrm{x}$ DPBS to remove any residual transfection reagent mix and replace with 2-3 $\mathrm{mL}$ of culture medium per well. Incubate under cell-specific optimal conditions until 72 hours posttransfection with changes of culture medium as required during this period.

\subsection{FACS for CRISPR-Edited Cells}

\subsubsection{Cell Preparation for FACS}

At 72 hours post-transfection, begin cell preparation for FACS. Trypsinise cells, wash with 1 $x$ DPBS and then stain with LIVE/DEAD Fixable Near-IR Dead Cell Stain Kit (Invitrogen) as per manufacturer's instructions. After staining, centrifuge cells and remove supernatant, then 
resuspend in $250 \mu \mathrm{L}$ of sterile autoMACS buffer. Also prepare and label a collection tube for each transfected sample (including negative control sample(s)) containing $250 \mu \mathrm{L}$ of $1 \mathrm{x}$ DPBS. Keep cells on ice until FACS is performed; for best results, perform FACS immediately once preparation is complete.

\subsubsection{FACS}

Perform FACS using an appropriate system which allows for the simultaneous selection of cells based on positivity for multiple fluorophores. We recommend the use of the BD FACSAria Fusion (BD Biosciences) or similar. Our methylation-editing system requires the selection of triple-positive cells, exhibiting simultaneous positivity for mTagBFP, sfGFP, and TagRFP657. Begin the FACS run by running the negative control sample, which allows accurate gates to be set for identifying positive cells.

Collect negative control cells for later analysis. Once gates are set, sort and collect transfected samples into separate $15 \mathrm{~mL}$ Falcon tubes. Collect only live cells which are triple-positive (i.e., positive for mTagBFP, sfGFP, and TagRFP657 simultaneously; Supplementary Figure S1). Once sorted, centrifuge cells and remove supernatant, then store at $-80^{\circ} \mathrm{C}$ until required for analysis.

\subsection{Targeted DNA Methylation Analysis for Confirmation of CRISPR Methylation Editing}

\subsubsection{DNA Preparation and Sequencing}

Evaluation of CRISPR-edited cell lines and confirmation of successful DNA methylation editing can be performed using a variety of targeted DNA methylation analysis methods [18, 19, 29, 30]. We recommend a high-throughput, next-generation sequencing-based approach such as methylation-specific Illumina MiSeq sequencing [31]. Such methods pair bisulfitespecific sequence amplification with deep sequencing to offer high-resolution assessment of methylation status at a very low cost per read. Begin preparation for targeted DNA methylation analysis by extracting genomic DNA from CRISPR-edited cells and performing bisulfite 
conversion. For cell numbers $>1.0 \times 10^{6}$, we recommend the use of an appropriate extraction method for genomic DNA and subsequent bisulfite conversion using the EZ DNA MethylationGold Kit (Zymo Research). For samples with cell numbers $<1.0 \times 10^{6}$, we recommend the use of EZ DNA Methylation-Direct Kit (Zymo Research), which allows for cell lysis and bisulfite conversion without an intermediate DNA purification step. Comprehensive methodologies for next-generation [31] and other targeted DNA methylation analysis methods [32] have been published previously and, therefore, will not be detailed further in this publication. For bisulfite-specific primer design, we recommend using the MethPrimer online design tool [33]. It should be noted that the size of the genomic locus interrogated via targeted DNA methylation analysis will be limited due to bisulfite treatment ( $<500 \mathrm{bp}$ ). Illumina universal adaptor sequences should be added to the 5' ends of the bisulfite-specific primers to facilitate sequencing on the Illumina MiSeq platform. Ordered oligonucleotide pairs should have the following sequences (where (N)22-25 is the bisulfite-specific primer sequence):

Forward oligonucleotide: $\quad$ 5'-ACGACGCTCTTCCGATCT(N)22-25-3'

Reverse oligonucleotide: 5'-CGTGTGCTCTTCCGATCT(N)22-25-3'

\subsubsection{Analysis of Targeted DNA Methylation Sequencing Data}

Processing and analysis of methylation-specific sequencing data requires specific software packages in order to convert raw sequencing data into a useable output. We briefly outline here the software used in our analysis pipeline, specifically for Illumina MiSeq sequencing data of our methylation-edited samples. Analysis of targeted DNA methylation sequencing data has been described in detail by other groups $[34,35]$.

Briefly, the paired sequencing data should first be merged into full-length reads using PEAR (Paired-End reAd mergeR) software [36]. Merged reads then undergo quality assessment using FastQC (Babraham Bioinformatics) and subsequent adaptor trimming using Trim Galore! (Version 0.5.0, Babraham Bioinformatics). Processed reads can then be uploaded into the BiQ Analyzer HT software [37] in FASTA format, which aligns reads to a specified genomic 
reference sequence. BiQ Analyzer HT generates binary methylation data for each aligned read, at each CpG dinucleotide within the amplicon (Supplementary Figure S2).

\section{6 gRNA Evaluation for On-Target Specificity and Off-Target Activity}

Despite the continuing advancement of publicly available prediction algorithms and design software, definitive evaluation of on- and off-target activities for unique gRNA molecules can be a valuable adjunct to DNA methylation-editing experiments. Without the need for cost- and labour-intensive whole-genome approaches, we describe an optional protocol for rapid gRNA evaluation using an active Cas9-based assay.

\subsubsection{Selection of Predicted Off-Target Loci and Primer Design}

First, select the top potential off-target sites for each respective gRNA. The most likely potential off-target loci are predicted via the in silico Benchling gRNA design process. We recommend selecting approximately twenty potential off-targets for evaluation. Subsequently, design a single set of primers for each off-target region using the NCBI Primer-BLAST online tool (https://www.ncbi.nlm.nih.gov/tools/primer-blast/index.cgi). Further, to establish the ontarget specificity of a respective gRNA, design a set of primers that include the respective gRNA target sequence. Each primer pair should be designed to flank the respective on- or offtarget locus plus $>100 \mathrm{bp}$ of sequence in either direction. Illumina universal adaptor sequences are added to the 5' terminus of each primer to facilitate Illumina MiSeq sequencing. Ordered oligonucleotide pairs should have the following sequences (where $(\mathrm{N}) 18-22$ is the primer sequence):

Forward oligonucleotide: $\quad$ 5'-ACGACGCTCTTCCGATCT(N)18-22-3'

Reverse oligonucleotide: 5'-CGTGTGCTCTTCCGATCT(N)18-22-3'

\subsection{2 gRNA and CRISPR-Cas9 Transfection}


Perform all steps of transfection and FACS as per sections 2.3 and 2.4; however, only transfect and sort a two-plasmid combination of gRNA (as prepared for methylation-editing) and active Cas9 (pSpCas9(BB)-2A-GFP). Perform genomic DNA extraction for each respective sample using an appropriate method. Do not perform bisulfite conversion here.

\subsubsection{Illumina MiSeq Sequencing}

Again, preparation of Illumina MiSeq libraries for next-generation sequencing are well documented [31]. A multiplexed library should be generated using isolated DNA from transfected cells post-FACS and containing amplicons for each of the respective on- and offtarget loci. The multiplexing strategy depends on the sequencing depth and the number of samples to be sequenced. Targeted amplicon sequencing libraries have low complexity, so PhiX genomic sequence is added to the sequencing pool to increase the diversity. The percentage of PhiX needed depends on the experimental design (i.e., the number of different regions to be sequenced). We obtained high coverage of 100x per sample together with minimum of 5\% PhiX DNA, allowing reproducible methylation analysis. The sequence read length is dependent on amplicon size and where the target site is located within the amplicon. If possible, the amplicon should be centered on the targeted site. Read length should be less than the amplicon length to avoid read through the primer sequence. Paired end chemistry is the preferred option as it allows a more precise edition call, especially for insertion or deletion (INDEL) detection, due to the overlap of the two strands. The sequence overlap between paired end reads should be at least 10 nucleotides. Once sequencing is complete, data can be processed using the following command line-based analysis workflow.

\subsubsection{Analysis of Sequencing Data}

First, sequencing reads with no mismatch compared to respective primers are demultiplexed using the grep command, then quality trimmed using fastq-mcf to include reads of $\geq Q 30$ only. Overhanging sequences outside of the primers are then trimmed off, and Illumina universal adaptors removed using fastq-mcf. Paired-end reads are then merged (mergedPE) using 
FLASH (Fast Length Adjustment of SHort reads) [38], not allowing outies. MergedPE reads are then made unique (UniqSeq) and the number of occurrences is counted for each unique sequence. UniqSeq reads are aligned to the reference sequence using Needle (i.e., the Needleman-Wunsch global alignment algorithm).

Aligned UniqSeq reads are parsed, and single nucleotide polymorphism (SNP) and INDEL information retrieved for the respective in-target sites. The in-target site for each respective UniqSeq refers to the locus at which the sgRNA anneals to the reference sequence. SNP or INDEL (non-reference nucleotides) occurring at the in-target site is used as a surrogate measure of Cas 9 activity and subsequent non-homologous end joining. True signal is distinguished from background noise via comparison against control samples (i.e., gRNA only and Cas9 only control samples). The below formula uses a conservative approach to estimate a background noise threshold using the maximum number of SNP or INDEL occurrences:

$$
\% \text { Background Noise }=\left(\frac{\text { Maximum Non Reference Nucleotide Occurences }}{\text { mergedPE Count }}\right) \times 100
$$

An ideal gRNA profile would, therefore, be supported by high occurrences of SNP and INDEL above background noise at on-target loci and with very low or no occurrences at off-target loci, respectively.

\section{Results and Discussion}

\subsection{Locus-Specific gRNA Design for Targeted Methylation-Editing}

Through careful gRNA design, we were able to achieve highly efficacious DNA methylation editing across three human melanoma cell lines, for a single target locus. Specifically, we targeted a 58 bp region of the $E B F 3$ gene promoter region (chr10:131,763,530-131,763,587; reference genome GRCh37/hg19), a gene that we have previously identified as a putative 'epigenetic driver' of melanoma metastasis [13]. This $58 \mathrm{bp}$ locus, containing a total of nine CpG sites, was identified from genome-scale discovery analyses as a potential transcriptional 
control region and is located -993 bp from the $E B F 3$ transcription start site. We selected a total of six gRNA molecules from initial in silico gRNA design (Supplementary Table S1), comprising three distinct pairs, targeted 32-34 bp upstream (gRNA \#1), corresponding to (gRNA \#2), and 74-76 bp downstream (gRNA \#3) of the target locus (Figure 2).

Preliminary experiments were performed in cell line NZM40 to assess the efficacy of demethylation via dCas9-SunTag and scFv-TET1CD, using gRNAs targeted to each of these respective loci. Interestingly, whilst gRNA(s) targeted to gRNA \#1 were able to induce successful demethylation at the target locus, no change in DNA methylation levels were observed for gRNA \#2 or gRNA \#3. Lack of response to targeting with gRNA \#2 may be due to one of two reasons: (1) direct binding of the dCas9-gRNA complex is known to result in steric blockade of at least the underlying 20-30 bp of sequence, thereby precluding the action of bound effector proteins; (2) dense methylation at the target locus, alongside tightly-packed heterochromatin, may in itself mechanically interfere with dCas9 binding [25, 39]. gRNA location \#3 may similarly be affected by inaccessibility to the DNA sequence, either at the point of dCas9 binding, or inaccessibility of bound effectors to target CpGs. Little is known with respect to the impacts of higher order chromatin organisation and topology on the action of DNA methylation editors; however, it is foreseeable that these factors have influenced the efficacy of our gRNAs for editing this particular locus. Indeed, achievable levels of editing efficiency and range of editing effect are variable between loci and editing systems, with ranges from as small as 8 bp to $1 \mathrm{~kb}$ from the PAM site currently reported $[18,40]$. Hence, the design of multiple gRNAs is useful when targeting a specific locus. On the basis of these findings, only gRNA(s) targeted to location gRNA \#1 were used for further methylation-editing experiments.

\subsection{Efficient Locus-Specific Editing of the EBF3 Gene Promoter}

Employing our aforementioned dCas9-SunTag system, in conjunction with scFv-DNMT3A and scFv-TET1CD effectors, respectively, we were able to induce both successful methylation 
and demethylation of the EBF3 target locus across multiple cell lines (Figure 3). We chose a panel of three human melanoma cell lines with variable levels of baseline methylation at our target locus. Only one group has previously demonstrated epigenetic editing in melanoma cells, limited to modifying histone phosphorylation of the A375 cell line [41].

We performed targeted DNA demethylation in cell lines WM115 and NZM40, which displayed a relative methylation decrease of $99.99 \%$ and $66.50 \%$, respectively (absolute methylation level change from $44.44 \%$ to $0.59 \%$ in WM1 15 , and $59.99 \%$ to $20.10 \%$ in NZM40). Targeted DNA methylation was induced at this same locus in CM150-Post, resulting in a relative methylation increase of $304.00 \%$ (absolute methylation change from $8.98 \%$ to $36.28 \%$ ). Relative methylation change between paired unedited and edited samples was calculated as:

\section{(Average Methylation Level of Unedited - Average Methylation Level of Edited) \\ Average Methylation Level of Unedited}

Biologically, these respective alterations in average DNA methylation level reflect a substantial change in the methylation status of the target locus between unedited and edited samples, which is consistent across all cell lines and technical replicates.

Interestingly, we observed more extensive changes in demethylation between WM115 and NZM40 cell lines, both relative and absolute. For cell line WM115, near-complete demethylation across the target locus was observed, whereas $20 \%$ of the methylation across this locus was preserved in NZM40. Previously, studies have demonstrated a difference in methylation-editing efficacy between cell lines due to disparities in transfection efficiency [42]. Using our approach, however, stringent FACS selection of only cells actively expressing our editing system, ensures that variations in transfection efficiency are adjusted for, providing directly comparable results between cell lines. Therefore, the reasons for this difference in our observed editing efficacy remain unclear, though an incubation time of 72 hours may have been insufficient to facilitate complete demethylation in NZM40. TET-mediated demethylation 
involves intermediate hydroxymethylation of cytosine residues, which is indistinguishable from $5 \mathrm{mC}$ via bisulfite conversion [43]. Hence, hydroxymethylated residues would be identified as $5 \mathrm{mC}$ during targeted sequencing. Slower-replicating cell lines would therefore undergo less complete $5 \mathrm{mC}$ loss via passive dilution in a given time frame and cells with lower expression of base excision repair-associated machinery may require additional time to achieve extensive methylation loss [44]. Each of these mechanisms may contribute to the differences observed in our cell lines, amongst other cell line-specific factors. With respect to gain of methylation in CM150-Post, the absolute mean methylation increase $(27.3 \%)$ was modest in comparison to our demethylation experiments at the same locus. This increase did, however, represent a large relative increase $(304.00 \%)$ in methylation, suggesting that it is likely to be significant from a biological standpoint. This result may reflect an innate resistance to gain of methylation at this locus for CM150-Post and it is plausible that active demethylation machinery may counteract the action of scFv-DNMT3A. Interestingly, Huang et al demonstrated a similar limit of editing efficacy with transient delivery of scFv-DNMT3A, though efficacy increased to around $80 \%$ over time when delivered via lentiviral transduction [19].

Overall, we report highly efficient and reproducible DNA methylation editing of a target $E B F 3$ promoter locus across a panel of human melanoma cell lines, using transient delivery of a dCas9-SunTag-based editing system.

\subsection{Highly Reproducible DNA Methylation Analysis using Targeted Sequencing}

Bland-Altman analysis (Figure 3b) was performed to assess the reproducibility of successive sequencing replicates, as performed on unedited samples for each cell line. Note that for this analysis, DNA methylation level is reported as a proportion between 0.0 to 1.0 , rather than as a percentage. This method compares the DNA methylation difference (y-axis) versus average (x-axis) between two successive unedited samples for each respective $\mathrm{CpG}$ site (i.e., $\mathrm{CpG}$ 1-9 across the target region), for each respective cell line. The bias values, which represent the 
average of plotted differences for each respective cell line, are very low, at -0.00693 (WM115), -0.00013 (NZM40), and -0.01720 (CM150-Post), respectively. Further, the 95\% limits of agreement are very narrow at -0.03535 to 0.02149 (WM115), -0.05039 to 0.05013 (NZM40), and -0.05726 to 0.02287 (CM150-Post). Our Bland-Altman analysis suggests that the inter-run variation is very low for any individual data point, with $<1 \%$ variation between independent technical replicates. Further, there are no identifiable trends or changes in variance with increasing average values, suggesting that there are no systematic differences between runs. In this context, the Bland-Altman analysis provides strong evidence that our sequencing results remain very consistent across independent replicates and, therefore, any observed alterations in DNA methylation level between unedited and edited samples are the result of targeted manipulation via our editing system.

\subsection{Effective Editing Window of the dCas9-SunTag System}

Though the primary objective of this study was to perform targeted manipulation of our previously identified 58 bp EBF3 locus, our DNA methylation analysis was designed to sequence a $285 \mathrm{bp}$ amplicon of the EBF3 promoter, containing a total of $34 \mathrm{CpG}$ sites (chr10:131,763,460-131,763,744; reference genome GRCh37/hg19). Concordant with previous findings, our dCas9-SunTag system was able to induce robust methylation change across at least our $285 \mathrm{bp}$ amplicon (Figure 4); effective methylation editing has previously been described up to $1 \mathrm{~kb}$ from the target site using dCas9-SunTag [18]. Indeed, dCas9-SunTag systems have a broader effective editing window than other described systems [29, 40, 45-47], due to the SunTag protein scaffold tail and capacity for effector multimerisation. Due to the position of our gRNA, CpG sites 1-4 within our sequenced amplicon are located within our gRNA target sequence. We observed demethylation at these four $\mathrm{CpG}$ sites, regardless of using a methylating (scFv-DNMT3A) or demethylating (scFv-TET1CD) effector construct. Again, this is consistent with previous findings which suggest that binding of the dCas9 module at the target site sterically prevents the activity of effector enzymes at underlying CpGs [25]; further, 
bound dCas9 may preclude the action of endogenous DNMTs, thereby promoting demethylation at these $\mathrm{CpG}$ residues.

Our dCas9-SunTag, scFv-TET1CD system demonstrated effective and consistent demethylation across the entire 285 bp amplicon (Figure 4a). For both NZM40 (mean relative demethylation $61.32 \%, \mathrm{SEM} \pm 2.50 \%$ ) and $\mathrm{WM} 115$ (mean relative demethylation $96.75 \%$, $\mathrm{SEM} \pm 1.08 \%$ ), relative levels of demethylation were remarkably consistent across all $34 \mathrm{CpG}$ sites, suggesting that our editing tool has reliable efficacy at $\mathrm{CpG}$ residues within a locus of this size. Interestingly, dCas9-SunTag, scFv-DNMT3A was more variable, with peak methylation increases observed around 25-120 bp and 180-190 bp from the PAM site (Figure 4b). Several $\mathrm{CpG}$ sites within the wider amplicon displayed a decrease in DNA methylation; however, this occurred exclusively at $\mathrm{CpG}$ sites with comparatively low baseline methylation levels $(<10 \%)$. As such, these methylation changes may carry less biological significance. The variations in editing efficacy observed with this tool may be secondary to structural differences between scFv-TET1CD and scFv-DNMT3A not permitting enzymatic activity at these residues, or due to an innate propensity for certain $\mathrm{CpG}$ sites to remain unmethylated. Overall, both tools were able to induce efficacious local methylation change within at least the constraints of our amplicon, with the exception of $\mathrm{CpG}$ sites underlying the bound dCas9 module, that underwent demethylation in a cell lines irrespective of which effector was used.

\subsection{Assessment of On- and Off-Target Activity for Targeted Editing}

Gold-standard methods for assessing off-target impacts include whole genome or ChIPbisulfite sequencing $[48,49]$. These methods, though thorough and accurate, are expensive and time-consuming. Comparatively, we employed a screening method that assesses specific loci with a high likelihood of off-target impacts, based on in silico prediction. This provided a rapid assessment of high-yield loci to give confidence in targeted editing results. As Cas9-binding at specific loci is gRNA-directed, INDEL formation at any given locus provides a surrogate measure of gRNA activity. Through selecting a smaller number of loci for assessment, this 
approach provides high yield off-target assessment without the need for more extensive, genome-wide approaches.

We perfomed a targeted gRNA assessment of gRNA location \#1, used for editing our EBF3 target region (Figure 5). Assessment of on-target activity, as well as activity at the top ten predicted off-target loci was performed. Two negative controls (Cas9 only; gRNA only) were included. Our gRNA displayed high on-target efficacy, with $90.48 \%$ of reads in the EBF3 target region containing INDELs above background noise; comparatively, $0.46 \%$ and $0.20 \%$ of the Cas9 and gRNA negative control samples contained INDELs above background noise, respectively. All three groups had very low rates of off-target INDEL generation at predicted off-target sites, with all $<1 \%$. Given the consistency of these INDEL rates with those of both negative control samples, any off-target INDELs are likely to be due to random mutation rather than direct effects of our editing system. For targeting of the EBF3 promoter, these data demonstrate that our selected gRNA(s) are highly efficacious at the desired locus, with minimal off-target activity. These findings are consistent with previous off-target assessments of dCas9SunTag systems, wherein whole-genome bisulfite sequencing results have demonstrated minimal off-target effects on methylation [19, 48].

Overall, we demonstrate a novel approach for gRNA assessment, which provides not only highyield and rapid screening for off-target effects of our editing system, but also accurately determines the on-target efficacy for a respective gRNA in a genuine in vitro context. With respect to our dCas9-SunTag system, these results provide confidence that with appropriate gRNA selection, a high level of on-target binding can be achieved, with minimal impacts on the wider DNA methylome.

\subsection{Design and Delivery Considerations for Editing System Selection}

Several CRISPR-based systems have now been described for DNA methylation editing, each providing unique advantages and limitations (Table 1). Our system is a variation of the SunTag 
system, originally described by Tanenbaum et al (2014) [50] and first adapted by Morita et al (2016) [18] for targeted DNA methylation editing. dCas9-SunTag systems are a widely used multimerisation approach which have shown high efficacy for both DNA methylation and demethylation, whilst displaying superior off-target profiles than other editing systems [18, 48]. SunTag systems also provide scope for the multimerisation of complementary effector constructs, such as TET1CD and VP64 for multi-level gene activation [51, 52].

Transient methods of construct delivery, including lipofection, provide a rapid and simple method of construct delivery and has been a favoured approach for DNA methylation editing to date $[18,29,30,39,40,45-47,53,54]$. Transient delivery methods have the benefit of simplicity and cost-effectiveness, allowing for straightforward, proof-of-concept experiments to be performed in a timely manner. Indeed, stable transfection methods, especially those which rely on viral delivery, require more specialised facilities and training to be performed, as well as typically longer time frames for the generation of transduced cell lines. As such, we prefer the use of a transient delivery approach in the first instance, supplemented with more stable approaches secondarily. The major advantage of stable delivery options, interestingly, would appear to be in efficiency of delivery for difficult cell lines rather than the stability of editing. In fact, numerous studies have now demonstrated that transient delivery of DNA methylation editing systems results in stable changes in methylation for long periods, with persistent changes observed for up to 50 days in replicating cells $[29,30,46]$. There is, however, some evidence that viral delivery may allow for more substantial targeted methylation changes in the context of dCas9-SunTag, scFv-DNMT3A [19].

To enhance the efficacy of our CRISPR-based tool in melanoma cell lines, we are currently optimising a lentiviral delivery system. Lentivirus-mediated transduction of our CRISPR-based editing system has the potential to complement our current approach via increasing the efficiency of targeted methylation editing and enabling the generation of stably expressing cell lines for further applications. 
Our own data support dCas9-SunTag as an effective system for manipulating DNA methylation. For the first time in human melanoma cell lines, we demonstrate highly efficacious, site-specific DNA methylation editing of the EBF3 gene promoter; we also demonstrate both targeted methylation and demethylation of a single locus. Paired with scFvDNMT3A, our dCas9-SunTag-based editing system was able to induce a substantial relative increase in DNA methylation at our target locus, in a lowly-methylated cell line. Likewise, our scFv-TET1CD effector was able to achieve up to near-complete demethylation, or unmethylation, in moderate to highly-methylated cell lines. Impacts on DNA methylation were consistent across our entire sequenced amplicon, providing support for the relatively broad editing window of this tool. Further, these effects were rapidly achieved with transient delivery our editing system. In addition to high levels of on-target efficacy, our off-target screening demonstrates that these tools act with high specificity, displaying minimal off-target activity. Together, these data highlight the efficacy, specificity and flexibility of our described system, which can be adapted for both targeted DNA methylation or demethylation using this fast and straighforward approach.

\subsection{Limitations and Troubleshooting}

Though optimized to suit the human melanoma cell lines WM115, CM150-Post and NZM40, this protocol may also be broadly applicable to other human cell lines for which Lipofectamine 3000 is an effective transfection reagent, particularly human melanoma cell lines. In our experience, electroporation proved an ineffective method for delivery of our editing system in these particular cell lines (data not shown) and so lipofection was chosen as our preferred method for transient construct delivery.

\subsubsection{Timing of Transfection}

The timing of cell culture in relation to transfection is crucial to maintain cell health, ensure adequate cell numbers are available, and to maximise transfectability. Different cell lines may 
require different growth times to achieve this, and so, a trial of growth from frozen stocks to adequately cultured cells is recommended prior to attempting transfection.

\subsubsection{DNA Quality and Total DNA Input}

Before beginning the first transfection, or when generating new batches of plasmid DNA for transfection, we recommend screening the isolated DNA for accuracy. As each of the constructs is produced using restriction cloning, this can easily be done via a restriction digest (e.g., RsrII/SpeI digest of an effector construct). Additionally, transfection is better tolerated when DNA purity is high and so a DNA purity assessment may be valuable prior to transfection (i.e., ensuring an adequate A260/A280 ratio).

Though optimized for the cell lines WM115, CM150-Post and NZM40 to 1500 ng total DNA per transfection reaction, optimal DNA input may vary between cell lines. If excessive cell toxicity is observed using $1500 \mathrm{ng}$ of total DNA, we recommend optimizing DNA input for each respective cell line.

\subsubsection{Exposure to Transfection Reagents}

Similar to total DNA input, Lipofectamine 3000 concentration and exposure time to transfection reagents are key factors which we have optimized for cell lines WM115, CM150Post and NZM40. Therefore, if cell toxicity is high, or if using different cell lines, we recommend optimizing Lipofectamine 3000 concentration per reaction and/or exposure time to transfection reagents. Each of these optimization experiments can be performed via the described transfection method and FACS selection, with subsequent analysis of the FACS data output to determine optimal transfection conditions. Note, a reverse-transfection method is described here as our group has had previous success using reverse transfection for these adherent cell lines. It would be reasonable to trial a standard transfection method in other cell lines, which has been demonstrated previously [13]. 


\subsubsection{Spectral Overlap Considerations for FACS}

If spectral overlap is predicted, then we recommend performing a FACS compensation experiment using cells transfected with each respective plasmid, individually. This allows the FACS system to be adjusted for inherent overlap between the emission spectra of fluorophores used in this editing system. To differentiate live versus dead or dying cells in the APC-Cy7 channel, we recommend using LIVE/DEAD Fixable Near-IR Dead Cell Stain, as per the manufacturer's instructions. If the spread of live versus dead cell populations shows excessive overlap, however, we recommend using a more dilute stain preparation (i.e., $1 \mu \mathrm{L}$ stain per 6$8 \mathrm{~mL} 1 \mathrm{x}$ DPBS). This may need to be modified with different cell lines.

\subsubsection{Transfection Efficiency}

Known limitations of our approach include relatively low transfection efficiency and, subsequently, low cell output post-FACS, largely due to the difficulties of co-transfecting three large plasmids simultaneously. Low cell outputs may present challenges for downstream applications, including investigation of changes in gene expression or chromatin organisation. For cell lines where cell numbers are insufficient for downstream analysis, alternative approaches should be considered, including other methods of transient system delivery. Without employing a stable delivery method such as viral packaging, substantial improvements in transfection efficiency may be difficult to achieve for some cell lines. Furthermore, the use of combined, or smaller, constructs could potentially mitigate the difficulties of co-transfecting multiple large plasmids [18]. When considering further analysis of samples with low cell numbers, we recommend the use of specialized kits for low cell input (e.g., EZ DNA Methylation-Direct Kit).

\section{Conclusions}

Here, we describe an effective protocol for targeted DNA methylation editing in human melanoma cell lines. We use transient lipofection as the delivery strategy for our dCas9SunTag-based editing system and provide a thorough workflow, including: system design; 
delivery; analysis; and a novel method for rapid off-target assessment. Although we focus on editing DNA methylation in the mammalian genome, the steps and principle of this protocol could be used to edit and study methylation of other model organisms, such as zebrafish [55, 56]. Employing this dCas9-SunTag-based editing approach, we performed highly efficacious manipulation of DNA methylation within the EBF3 promoter, across multiple cell lines, inducing substantial levels of targeted methylation and demethylation, with minimal off-target effects.

\section{Data Availability Statement}

The original contributions presented in the work are included in the article. Further inquiries can be directed to the corresponding authors.

\section{Author Contributions}

Conceptualization, A.C, R.J.W; methodology, G.G, A.C, R.J.W; software, J.S, R.B, R.W, A.U, G.G and R.J.W; validation, J.S, R.B and R.W; formal analysis, J.S, R.B, R.W, A.U, G.G, R.D and R.J.W; investigation, J.S, R.B, R.W, A.U, R.D, R.J.W; resources, R.D, M.R.E, A.C and R.J.W; data curation, J.S, R.B, R.W, A.U, G.G and R.J.W; writing-original draft preparation, J.S; writing — review and editing, all authors; visualization, J.S, R.B, R.W, A.U, G.G and R.J.W; supervision, A.C, R.J.W and M.R.E; project administration, A.C and R.J.W; funding acquisition, A.C. All authors have read and agreed to the published version of the manuscript.

\section{Conflicts of Interest}

The authors declare no conflict of interest.

\section{Funding}

This research was funded by a Marsden Fast Start Fund (RSNZ), grant number 17-UOO-240, a Rutherford Discovery Fellowship to Aniruddha Chatterjee and internal funds from University of Otago and the APC was funded by the University of Otago. 


\section{Acknowledgments}

We would like to acknowledge Michelle Wilson (Flow Cytometry Technician, University of Otago) for her expertise and assistance in performing FACS, Dr Jisha Antony for her helpful discussion of CRISPR experiments, and Professor Ian Morison and Dr Peter Stockwell for their support in our work on methylation editing.

\section{References}

1. Jones, P.A. (2012) Functions of DNA methylation: islands, start sites, gene bodies and beyond. Nature Reviews Genetics 13 (7), 484.

2. Baylin, S.B. (2005) DNA methylation and gene silencing in cancer. Nature Clinical Practice Oncology 2, S4.

3. Chatterjee, A. et al. (2018) Marked Global DNA Hypomethylation Is Associated with Constitutive PD-L1 Expression in Melanoma. iScience 4, 312-325.

4. Chatterjee, A. et al. (2018) Epigenetic drivers of tumourigenesis and cancer metastasis. Semin Cancer Biol 51, 149-159.

5. Witte, T. et al. (2014) Pan-cancer patterns of DNA methylation. Genome Med 6 (8), 1-18. 6. Miranda, T.B. and Jones, P.A. (2007) DNA methylation: the nuts and bolts of repression. J Cell Physiol 213 (2), 384-390.

7. Smith, J. et al. (2020) Promoter DNA Hypermethylation and Paradoxical Gene Activation. Trends in Cancer.

8. Guilleret, I. et al. (2002) Hypermethylation of the human telomerase catalytic subunit (hTERT) gene correlates with telomerase activity. Int J Cancer 101 (4), 335-341.

9. Castelo-Branco, P. et al. (2013) Methylation of the TERT promoter and risk stratification of childhood brain tumours: an integrative genomic and molecular study. The lancet oncology 14 (6), 534-542.

10. Bert, Saul A. et al. (2013) Regional Activation of the Cancer Genome by Long-Range Epigenetic Remodeling. Cancer Cell 23 (1), 9-22. 
11. Takasawa, K. et al. (2018) DNA hypermethylation enhanced telomerase reverse transcriptase expression in human-induced pluripotent stem cells. Hum Cell 31 (1), 78-86.

12. Spainhour, J.C.G. et al. (2019) Correlation patterns between DNA methylation and gene expression in The Cancer Genome Atlas. Cancer Inform 18, 1176935119828776.

13. Chatterjee, A. et al. (2017) Genome-wide methylation sequencing of paired primary and metastatic cell lines identifies common DNA methylation changes and a role for EBF3 as a candidate epigenetic driver of melanoma metastasis. Oncotarget 8 (4), 6085.

14. Rodger, E.J. et al. (2019) Characterisation of DNA methylation changes in EBF3 and TBC1D16 associated with tumour progression and metastasis in multiple cancer types. Clin Epigenetics $11(1), 114$.

15. Greenberg, M.V.C. and Bourc'his, D. (2019) The diverse roles of DNA methylation in mammalian development and disease. Nature reviews Molecular cell biology 20 (10), 590-607. 16. Urbano, A. et al. (2019) Gene-Specific Targeting of DNA Methylation in the Mammalian Genome. Cancers (Basel) 11 (10), 1515.

17. Nakamura, M. et al. (2021) CRISPR technologies for precise epigenome editing. Nat Cell Biol 23 (1), 11-22.

18. Morita, S. et al. (2016) Targeted DNA demethylation in vivo using dCas9-peptide repeat and scFv-TET1 catalytic domain fusions. Nat Biotechnol 34 (10), 1060.

19. Huang, Y.-H. et al. (2017) DNA epigenome editing using CRISPR-Cas SunTag-directed DNMT3A. Genome Biol 18 (1), 176.

20. Yang, X. et al. (2015) Systematic DNA methylation analysis of multiple cell lines reveals common and specific patterns within and across tissues of origin. Hum Mol Genet 24 (15), 4374-4384.

21. Rodger, E.J. et al. (2021) Comparison of Global DNA Methylation Patterns in Human Melanoma Tissues and Their Derivative Cell Lines. Cancers (Basel) 13 (9), 2123.

22. Anders, C. et al. (2014) Structural basis of PAM-dependent target DNA recognition by the Cas9 endonuclease. Nature 513 (7519), 569. 
23. Lo, C.-L. et al. (2017) Epigenetic Editing of Ascll Gene in Neural Stem Cells by Optogenetics. Sci Rep 7 (1), 42047.

24. Pellegrini, R. How to Synthesize your gRNAs for CRISPR.

25. Stepper, P. et al. (2016) Efficient targeted DNA methylation with chimeric dCas9Dnmt3a-Dnmt3L methyltransferase. Nucleic Acids Res 45 (4), 1703-1713.

26. Liu, X.S. et al. (2016) Editing DNA methylation in the mammalian genome. Cell 167 (1), 233-247.

27. Ran, F.A. et al. (2013) Genome engineering using the CRISPR-Cas9 system. Nat Protoc 8 (11), 2281.

28. Franco, A.V. et al. (2001) The role of NF- $\kappa B$ in TNF-related apoptosis-inducing ligand (TRAIL)-induced apoptosis of melanoma cells. The Journal of Immunology 166 (9), 53375345.

29. Vojta, A. et al. (2016) Repurposing the CRISPR-Cas9 system for targeted DNA methylation. Nucleic Acids Res 44 (12), 5615-5628.

30. Nuñez, J.K. et al. (2021) Genome-wide programmable transcriptional memory by CRISPRbased epigenome editing. Cell 184 (9), 2503-2519.

31. Masser, D.R. et al. (2015) Targeted DNA methylation analysis by next-generation sequencing. Journal of visualized experiments: JoVE (96).

32. Cheishvili, D. et al. (2017) Targeted DNA methylation analysis methods. In Epigenetics and Gene Expression in Cancer, Inflammatory and Immune Diseases, pp. 33-50, Springer.

33. Li, L.-C. and Dahiya, R. (2002) MethPrimer: designing primers for methylation PCRs. Bioinformatics 18 (11), 1427-1431.

34. Chatterjee, A. et al. (2017) Tools and strategies for analysis of genome-wide and genespecific DNA methylation patterns. In Oral Biology, pp. 249-277, Springer.

35. Almomani, S.N. et al. (2021) Identification and validation of DNA methylation changes in pre-eclampsia. Placenta.

36. Zhang, J. et al. (2013) PEAR: a fast and accurate Illumina Paired-End reAd mergeR. Bioinformatics 30 (5), 614-620. 
37. Lutsik, P. et al. (2011) BiQ Analyzer HT: locus-specific analysis of DNA methylation by high-throughput bisulfite sequencing. Nucleic Acids Res 39 (suppl_2), W551-W556.

38. Magoč, T. and Salzberg, S.L. (2011) FLASH: fast length adjustment of short reads to improve genome assemblies. Bioinformatics 27 (21), 2957-2963.

39. Devesa-Guerra, I. et al. (2020) DNA Methylation Editing by CRISPR-guided Excision of 5-Methylcytosine. J Mol Biol 432 (7), 2204-2216.

40. Xiong, T. et al. (2017) Targeted DNA methylation in human cells using engineered dCas9methyltransferases. Sci Rep 7 (1), 6732.

41. Li, J. et al. (2021) Programmable human histone phosphorylation and gene activation using a CRISPR/Cas9-based chromatin kinase. Nature Communications 12 (1), 896.

42. Choudhury, S.R. et al. (2016) CRISPR-dCas9 mediated TET1 targeting for selective DNA demethylation at BRCA1 promoter. Oncotarget 7 (29), 46545.

43. Huang, Y. et al. (2010) The behaviour of 5-hydroxymethylcytosine in bisulfite sequencing. PLoS One 5 (1), e8888.

44. Kohli, R.M. and Zhang, Y. (2013) TET enzymes, TDG and the dynamics of DNA demethylation. Nature 502 (7472), 472-479.

45. Xu, X. et al. (2016) A CRISPR-based approach for targeted DNA demethylation. Cell discovery 2,16009 .

46. Lei, Y. et al. (2017) Targeted DNA methylation in vivo using an engineered dCas9-MQ1 fusion protein. Nature communications 8, 16026.

47. Lu, A. et al. (2019) Reprogrammable CRISPR/dCas9-based recruitment of DNMT1 for site-specific DNA demethylation and gene regulation. Cell Discovery 5 (1).

48. Pflueger, C. et al. (2018) A modular dCas9-SunTag DNMT3A epigenome editing system overcomes pervasive off-target activity of direct fusion dCas9-DNMT3A constructs. Genome Res, gr-233049.

49. Bao, X.R. et al. (2021) Tools for experimental and computational analyses of off-target editing by programmable nucleases. Nat Protoc 16 (1), 10-26. 
50. Tanenbaum, M.E. et al. (2014) A protein-tagging system for signal amplification in gene expression and fluorescence imaging. Cell 159 (3), 635-646.

51. Morita, S. et al. (2020) Synergistic Upregulation of Target Genes by TET1 and VP64 in the dCas9-SunTag Platform. Int J Mol Sci 21 (5), 1574.

52. Marx, N. et al. (2021) Enhanced targeted DNA methylation of the CMV and endogenous promoters with dCas9-DNMT3A3L entails distinct subsequent histone modification changes in CHO cells. Metabolic Engineering 66, 268-282.

53. Taghbalout, A. et al. (2019) Enhanced CRISPR-based DNA demethylation by Casilio-MEmediated RNA-guided coupling of methylcytosine oxidation and DNA repair pathways. Nature Communications 10 (1).

54. Alexander, J. et al. (2019) Concurrent genome and epigenome editing by CRISPRmediated sequence replacement. BMC Biol 17 (1), 1-13.

55. Chatterjee, A. et al. (2016) Sex differences in DNA methylation and expression in zebrafish brain: a test of an extended 'male sex drive'hypothesis. Gene 590 (2), 307-316.

56. Chatterjee, A. et al. (2014) Base-resolution DNA methylation landscape of zebrafish brain and liver. Genomics Data 2, 342-344.

\section{Appendix A}

Reagents:

- $\quad$ Human melanoma cell lines to be transfected (e.g. NZM40, WM115, CM150-Post)

- $\quad$ Appropriate cell culture medium for chosen cell line(s)

- $\quad 0.05 \%$ Trypsin-EDTA

- $\quad$ BsmBI restriction endonuclease and NEBuffer 3.1 1 TM $($ New England Biolabs)

- $\quad$ Shrimp alkaline phosphatase (rSAP)

- $\quad$ DNA Clean and Concentrator-5 Kit (Zymo Research), or equivalent

- $\quad$ T4 DNA Ligase and 10x T4 DNA Ligase Buffer (ThermoFisher Scientific)

- $\quad$ Pre-prepared CRISPR-methylation plasmids

- $\quad$ LB agar and broth for propagation of pre-prepared CRISPR-methylation plasmids 
- $\quad$ Appropriate antibiotic for plasmid selection (e.g. $100 \mu \mathrm{g} / \mathrm{mL}$ ampicillin)

- $\quad$ Plasmid isolation system (e.g. GenCatch ${ }^{\mathrm{TM}}$ Plasmid Plus DNA Maxiprep Kit (Epoch Life Science Inc))

- $\quad$ Opti-MEM serum-free medium (Invitrogen)

- $\quad$ Lipofectamine 3000 reagent and P3000 reagent (Invitrogen)

- 1 x Dulbecco's phosphate buffered saline (DPBS)

- $\quad$ Sterile autoMACS buffer ( 1 x DPBS + 1\% FCS + 2 mM EDTA)

- $\quad$ Ice for keeping cells pre- and post-fluorescence-activated cell sorting (FACS)

- $\quad$ DNA isolation kit for transfected FACS-selected cells

- $\quad$ Bisulfite conversion kit (e.g. EZ DNA Methylation-Gold Kit (Zymo Research)

- $\quad$ For low cell numbers, the combined DNA isolation and bisulfite conversion kit (e.g. EZ DNA Methylation-Direct Kit (Zymo Research))

- Reagents for targeted DNA methylation analysis (see 2.5 Targeted DNA Methylation Analysis for Confirmation of CRISPR-Editing)

Equipment:

- Access to the Benchling online platform for gRNA Design (http://benchling.com)

- Cell culture facilities and incubator with appropriate conditions for chosen cell line(s)

- $\quad$ Cell culture flasks

- $\quad$ Tube(s) for cell resuspension (e.g. $15 \mathrm{~mL}$ or $50 \mathrm{~mL}$ Falcon conical centrifuge tubes)

- $\quad$ Centrifuge suitable for $15 \mathrm{~mL}$ or $50 \mathrm{~mL}$ Falcon tubes

- $\quad$ Cell counting apparatus (e.g. haemocytometer)

- $\quad$ Shaking incubator suitable for mid-scale $(200-500 \mathrm{~mL})$ bacterial cultures

- $\quad 1.5 \mathrm{~mL}$ microcentrifuge tubes

- 6-well cell culture plates

- $\quad 15 \mathrm{~mL}$ Falcon tubes for FACS cell preparation and collection 
bioRxiv preprint doi: https://doi.org/10.1101/2021.10.12.463855; this version posted October 12,2021 . The copyright holder for this preprint (which was not certified by peer review) is the author/funder, who has granted bioRxiv a license to display the preprint in perpetuity. It is made available under aCC-BY-ND 4.0 International license.

- $\quad$ FACS system with capacity for sorting cells which are positive for at least three fluorophores simultaneously (e.g. BD FACSAria Fusion (BD Biosciences))

- $\quad$ Equipment for targeted DNA methylation analysis (see 2.5 Targeted DNA Methylation Analysis for Confirmation of CRISPR-Editing)

- $\quad$ Access to the NCBI Primer-BLAST online tool for optional gRNA assessment experiments (https://www.ncbi.nlm.nih.gov/tools/primer-blast/index.cgi)

\section{Tables}

Table 1. Pioneering studies in the development of CRISPR-based DNA methylation editing systems for mammalian cells.

\begin{tabular}{|c|c|c|c|c|c|}
\hline Group & System & Description & Features & Delivery & Stability \\
\hline $\begin{array}{c}\text { Vojta et al } \\
(2016) \\
{[29]}\end{array}$ & dCas9-Effector & $\begin{array}{l}\text { Described the first dCas } 9 \\
\text { fusion with DNMT3A for } \\
\text { targeted methylation } \\
\text { editing in a } \sim 35 \text { bp wide } \\
\text { region for the } B A C H 2 \text { and } \\
\text { IL6ST loci, with } \\
\text { associated gene } \\
\text { expression change }\end{array}$ & $\begin{array}{l}\text { Simple design } \\
\text { Higher off-target } \\
\text { activity } \\
\text { Moderate editing } \\
\text { window } \\
(\sim 150 \mathrm{bp})\end{array}$ & Lipofection & $\begin{array}{c}\text { Peak increase } \\
\text { in } \\
\text { methylation } \\
\text { at day 6-7 } \\
\text { post- } \\
\text { transfection; } \\
\text { persistent } \\
\text { increases } \\
\text { observed to } \\
\text { at least } 42 \\
\text { days }\end{array}$ \\
\hline $\begin{array}{c}\mathrm{Xu} \text { et al } \\
(2016)[45]\end{array}$ & $\begin{array}{c}\text { dCas9, } \\
\text { Tet1-MS2 }\end{array}$ & $\begin{array}{c}\text { Designed a Effector-MS2 } \\
\text { and dCas9 with modified } \\
\text { gRNAs for effector } \\
\text { multimerisation }\end{array}$ & \begin{tabular}{|c|} 
Allows multimerisation \\
of effector constructs \\
Lower editing efficacy \\
Moderate editing \\
window \\
( $>100$ but $<300$ bp; no \\
editing within 100 bp of \\
gRNA) \\
\end{tabular} & $\begin{array}{c}\text { Polyethylenimine; } \\
\text { Lipofection }\end{array}$ & \begin{tabular}{|c|} 
mRNA \\
expression \\
change \\
peaked 4 \\
days post- \\
transfection \\
only
\end{tabular} \\
\hline \begin{tabular}{|} 
Morita et al \\
$(2016)[18]$
\end{tabular} & dCas9-SunTag & \begin{tabular}{|} 
Adapted the SunTag \\
protein scaffold for \\
multimerised DNA \\
methylation editing, \\
increasing the amino acid \\
linker length to $22 \mathrm{nt}$ to \\
achieve demethylation \\
efficacies of $>90 \%$ both \\
in vitro and in vivo
\end{tabular} & \begin{tabular}{|} 
Allows multimerisation \\
of effector constructs \\
Higher efficacy than \\
dCas9-Effector, -MS2, - \\
MQ1, -sMTase systems \\
Low off-target activity \\
Broader editing window \\
$(\leq 1 \mathrm{~kb})$
\end{tabular} & Lipofection & $\mathrm{N} / \mathrm{A}$ \\
\hline \begin{tabular}{|c|} 
Lei et al \\
$(2017)[46]$
\end{tabular} & dCas9-MQ1 & \begin{tabular}{|c|} 
Fused dCas9 with an \\
engineered prokaryotic \\
CpG DNA \\
methyltransferase "MQ1" \\
derived from Mollicutes \\
spiroplasma (M.SssI), \\
strain MQ1 \\
\end{tabular} & \begin{tabular}{|} 
Quicker onset of \\
effective methylation \\
change $(24 \mathrm{hrs})$ \\
Narrow editing window \\
$(\sim 30-50 \mathrm{bp})$
\end{tabular} & Lipofection & $\geq 3$ weeks \\
\hline
\end{tabular}




\begin{tabular}{|c|c|c|c|c|c|}
\hline \begin{tabular}{|l|} 
Xiong et al \\
$(2017)[40]$
\end{tabular} & dCas9-sMTase & \begin{tabular}{|} 
Developed a targeted, \\
"split methyltransferase" \\
derived from $M . S s s I$ \\
where the two split \\
components bind at the \\
target CpG site
\end{tabular} & \begin{tabular}{|c|} 
Low off-target activity \\
Narrow editing window \\
$(8-25 \mathrm{bp})$
\end{tabular} & Lipofection & $\mathrm{N} / \mathrm{A}$ \\
\hline \begin{tabular}{|} 
Taghbalout \\
et al (2019) \\
{$[53]$}
\end{tabular} & Casilio-ME & $\begin{array}{l}\text { Adapted the Casilio } \\
\text { platform for methylation } \\
\text { editing, providing co- } \\
\text { delivery of TET1 and } \\
\text { BER-associated proteins } \\
\text { GADD45A or NEIL2 }\end{array}$ & $\begin{array}{c}\text { Allows multimerisation } \\
\text { of effector constructs } \\
\text { Comparable or higher } \\
\text { efficacy than SunTag } \\
\text { systems } \\
\text { Low off-target activity }\end{array}$ & Lipofection & $\mathrm{N} / \mathrm{A}$ \\
\hline $\begin{array}{c}\text { Lu et al } \\
(2019)[47]\end{array}$ & dCas9-R2 & $\begin{array}{c}\text { Developed a dCas9-R2 } \\
\text { module which specifically } \\
\text { binds and inhibits the } \\
\text { action of endogenous } \\
\text { DNMT1 to prevent local } \\
\text { DNA methylation at a } \\
\text { target locus }\end{array}$ & \begin{tabular}{|} 
Narrow editing window \\
$(<100 \mathrm{bp})$ \\
No requirement for \\
exogenous effectors \\
Similar efficacy to \\
dCas9, Tet1-MS2
\end{tabular} & Lipofection & $\begin{array}{l}\text { Peaked at } 7 \\
\text { days post- } \\
\text { transfection }\end{array}$ \\
\hline \begin{tabular}{|c|} 
Alexander \\
et al $(2019)$ \\
{$[54]$}
\end{tabular} & $\begin{array}{c}\text { Dual Cas9, } \\
\text { Template }\end{array}$ & $\begin{array}{c}\text { Excised a } 1120 \text { bp CGI } \\
\text { from the } H P R T 1 \text { promoter } \\
\text { using dual gRNA-guided } \\
\text { Cas9 modules and } \\
\text { replaced with either } \\
\text { completely methylated or } \\
\text { unmethylated fragments } \\
\text { via NHEJ }\end{array}$ & \begin{tabular}{|c|} 
Ensures complete \\
de/methylation \\
NHEJ-mediated \\
insertion has low \\
efficiency $(<1 \%)$ \\
Prone to repair-induced \\
INDEL formation
\end{tabular} & Lipofection & $\mathrm{N} / \mathrm{A}$ \\
\hline \begin{tabular}{|c|} 
Devesa- \\
Guerra et al \\
$(2020)[39]$
\end{tabular} & dCas9-ROS1 & $\begin{array}{c}\text { Described a dCas9- } \\
\text { Effector using the plant- } \\
\text { specific DNA glycosylase } \\
\text { ROS1, which directly } \\
\text { excises } 5 \mathrm{mC}\end{array}$ & \begin{tabular}{|} 
Higher gene reactivation \\
efficacy than dCas9- \\
Effector fusions alone; \\
ineffective at fully \\
methylated loci \\
Modest impact on \\
measured methylation \\
levels
\end{tabular} & Lipofection & $\mathrm{N} / \mathrm{A}$ \\
\hline $\begin{array}{l}\text { Nuñez et al } \\
(2021)[30]\end{array}$ & CRISPRoff & $\begin{array}{c}\text { Developed a novel dCas9- } \\
\text { Dnmt3A-3L-ZNF10 } \\
\text { KRAB fusion } \\
\text { (CRISPRoff) to induce } \\
\text { heritable, persistent gene } \\
\text { silencing }\end{array}$ & \begin{tabular}{|c|} 
High efficacy of \\
methylation editing \\
Applicable to genome- \\
wide screening \\
Stable editing effect \\
despite transient \\
delivery \\
Non-isolated effect of \\
multiple enzymes (e.g., \\
DNMTs plus KRAB)
\end{tabular} & $\begin{array}{l}\text { Lipofection; } \\
\text { Nucleofection; } \\
\text { Lentiviral }\end{array}$ & $\begin{array}{c}\text { Construct } \\
\text { expression } \\
\text { lost at } 10 \\
\text { days; } \\
\text { methylation } \\
\text { change peaks } \\
\text { at } 9 \text { days, } \\
\text { stable for at } \\
\text { least } 30-50 \\
\text { days post- } \\
\text { transfection }\end{array}$ \\
\hline \begin{tabular}{|c|} 
Smith et al \\
(2021) \\
(Current \\
Manuscript)
\end{tabular} & dCas9-SunTag & $\begin{array}{c}\text { Applied dCas9-SunTag } \\
\text { with scFv-TET1CD and } \\
\text { scFv-DNMT3A effectors } \\
\text { to manipulate EBF3 } \\
\text { promoter methylation in } \\
\text { melanoma }\end{array}$ & \begin{tabular}{|} 
Achieved near-complete \\
demethylation and high \\
methylation \\
Comprehensive \\
workflow for \\
methylation-editing and \\
downstream analyses \\
\end{tabular} & Lipofection & $\mathrm{N} / \mathrm{A}$ \\
\hline
\end{tabular}


Supplementary Table S1. Forward and reverse oligonucleotide sequences for $E B F 3$ promoter gRNAs.

\begin{tabular}{|c|c|c|c|}
\hline Location & Target Strand & Forward/Reverse & Sequence \\
\hline \multirow{4}{*}{ gRNA Location \#1 } & \multirow{2}{*}{ Sense } & Forward & CACCGAAAAACCAAGCGGACGCCGC \\
\hline & & Reverse & AAACGCGGCGTCCGCTTGGTTTTTC \\
\hline & \multirow{2}{*}{ Antisense } & Forward & CACCGCGCGGCGTCCGCTTGGTTTT \\
\hline & & Reverse & AAACAAAACCAAGCGGACGCCGCGC \\
\hline \multirow{4}{*}{ gRNA Location \#2 } & \multirow{2}{*}{ Sense } & Forward & CACCGCGGCGCGCGGCTTCCCGACC \\
\hline & & Reverse & AAACGGTCGGGAAGCCGCGCGCCGC \\
\hline & \multirow{2}{*}{ Antisense } & Forward & CACCGGCGCGCTCACCCGGGTCCGG \\
\hline & & Reverse & AAACCCGGACCCGGGTGAGCGCGCC \\
\hline \multirow{4}{*}{ gRNA Location \#3 } & \multirow{2}{*}{ Sense } & Forward & CACCGCAAAGGACGTCTGCGCGACA \\
\hline & & Reverse & AAACTGTCGCGCAGACGTCCTTTGC \\
\hline & \multirow{2}{*}{ Antisense } & Forward & CACCGCGTGTCGCGCAGACGTCCTT \\
\hline & & Reverse & AAACAAGGACGTCTGCGCGACACGC \\
\hline
\end{tabular}

${ }^{a}$ Added 5 ' guaninge residues are highlighted in red; overhang sequences for cloning are highlighted in bold.

\section{Figure Legends}

Figure 1. Overview of our CRISPR-based methylation editing approach. (a) Components of the CRISPR-methylation editing system. Shown are the three broad components of our editing system: a CRISPR-dCas9 construct for locus-specific targeting with an associated SunTag protein scaffold; a gRNA construct including a unique target sequence (red); and an effector protein construct (blue) with associated $\mathrm{scFv}$ domain (purple) for binding to the SunTag scaffold and tagged sfGFP fluorophore (green circle). (b) Structure and size of each plasmid is shown, corresponding to each of the respective constructs. (c) System components are cloned, propagated, and isolated as plasmid DNA for transfection into cultured cells, which provides transient delivery of each construct simultaneously to induce in vitro methylation or demethylation. A unique gRNA guides the CRISPR-dCas9-SunTag construct to bind at the target locus. Subsequently, multiple effector proteins bind to each respective GCN4 domain of the SunTag scaffold, wherein they can induce methylation change at surrounding $\mathrm{CpG}$ dinucleotides. Through modifying the effector enzyme, this system can be adapted for targeted DNA methylation or demethylation as desired. Post-transfection, cells positive for the expression of all components of the editing system are collected via fluorescence-activated cell 
sorting (FACS) and used for downstream analyses, including targeted DNA methylation sequencing. Adapted with permission from Urbano et al (2019) [16].

Figure 2. Variable efficacy of gRNAs designed to target the $E B F 3$ promoter. Shown are the three locations of the EBF3 promoter, for which gRNAs were designed via in silico selection. For each location, two gRNA were designed, targeting the sense and antisense strands, respectively. Methylation levels within our target 58 bp locus are reported for cell line NZM40 in unedited versus edited samples, for each of the gRNA locations targeted. The position of each gRNA location with respect to the target locus is also shown.

Figure 3. (a) Targeted DNA methylation and demethylation of the $E B F 3$ promoter region across three human melanoma cell lines. Average levels of DNA methylation $(\%) \pm$ SEM (standard error of the mean; error bars) are shown for each of the cell lines WM115, NZM40 and CM150-Post, respectively, for both unedited and methylation-edited samples (absolute values per sample as shown). Note, no error bars are shown for NZM40 unedited sample as the SEM is too small to represent graphically. Active demethylation is demonstrated in WM115 and NZM40, whilst active methylation is demonstrated in CM150-Post. The relative level of DNA methylation change from unedited to edited is displayed as shown (\%). Corresponding heatmap representations are shown for each sample, each of which was generated using 500 randomly selected sequencing reads to illustrate the methylation status of each $\mathrm{CpG}$ site within the target region; methylated (red), unmethylated (blue), or unaligned (white). (b) BlandAltman analysis of replicate WM115, NZM40 and CM150-Post unedited samples per CpG. Plotted results of the Bland-Altman analysis between the corresponding per $\mathrm{CpG}$ methylation levels of successive replicate samples. Two technical replicates of unedited samples were analysed for each cell line as shown.

Figure 4. (a) Targeted DNA demethylation of the $E B F 3$ promoter region for NZM40 and WM115 cell lines. Mean DNA methylation levels between unedited (black) and edited (red) 
samples are plotted for each of the $34 \mathrm{CpG}$ sites within the 285 bp sequenced amplicon of the EBF3 promoter. Mean per $\mathrm{CpG}$ methylation levels are shown by relative genomic position (top), with the gRNA binding site (orange) and target region (light green) highlighted as shown. Absolute DNA methylation change (bottom left) and relative demethylation (\%) (bottom right; teal) are plotted per $\mathrm{CpG}$ for the entire amplicon. Mean relative demethylation across all 34 CpGs (teal line) and standard error of the mean (SEM; dotted teal line) are shown. (b) Targeted methylation of the $E B F 3$ promoter region for cell line CM150-Post. Mean DNA methylation levels between unedited and edited samples are plotted for each of the $34 \mathrm{CpG}$ sites by relative genomic position (top). Absolute DNA methylation change (bottom left) and relative demethylation (proportion) (bottom right) are plotted per $\mathrm{CpG}$ for the entire amplicon. Mean relative methylation across all $34 \mathrm{CpGs}$ and SEM are plotted as shown.

Figure 5. (a) Rapid screening method for on- and off-target assessment of unique gRNA constructs. The gRNA of interest and active Cas 9 construct are transfected into cell lines. Directed by the gRNA, Cas9 binds to genomic loci and induces double-stranded cleavage of the DNA molecule, which is subsequently repaired via non-homologous end joining (NHEJ). NHEJ introduces insertions or deletions (INDELs; orange) at the repaired locus which can be detected via sequencing, providing a surrogate for gRNA + Cas9 activity at any given locus. The on-target locus and top ten off-target loci are then sequenced via targeted high-throughput sequencing. (b) Assessment of on- and off-target activity for the gRNA used to successfully edit $E B F 3$ promoter methylation. Percentage of sequenced reads containing INDELs provides the surrogate measure of activity at each locus; data from off-target loci are combined as shown. Our gRNA shows very high on-target efficacy of INDEL generation and minimal off-target impacts for the top ten predicted off-target loci. Cas9 only and gRNA only controls display minimal on- or off-target effects.

Supplementary Figure S1. FACS for CRISPR-edited cells. (a) Gating setup using DNA-free negative control samples. Shown is the use of a DNA-free negative control sample to set 
accurate gates for fluorophore-positive (i.e., transfected) cells. Gates are set for each respective channel to ensure that only genuine fluorophore-positive cells will be sorted during FACS. (b) Example of FACS for CRISPR-edited NZM40 human melanoma cells. Shown is an example of the sequential gating strategy for sorting triple-positive transfected cells in practice, which selects sequentially for: the true cell population; live cells only; cells expressing TagRFP657; cells also expressing mTagBFP; cells also expressing sfGFP. Hence, all cells which are eventually sorted into the final collected population are live, triple-positive cells.

Supplementary Figure S2. BiQ Analyser HT processing during the analysis of targeted methylation sequencing data. (a) Example output results.tsv file (opened in Microsoft Excel) summarising the results of sequence alignment for a particular demultiplexed sample with 34 CpG sites interrogated in BiQ Analyser HT. This provides all information for a particular read following alignment and calculates multiple parameters to assess the success of read alignment. The binary methylation output is also shown $(1=$ methylated; $0=$ unmethylated; $\mathrm{x}=$ unaligned). As shown, the first four reads in this example have very low alignment scores, and these will be removed by further analyses (alignment score threshold $\geq 1000$ ). (b) Example methylation heatmap output visually displaying binary methylation data for $34 \mathrm{CpG}$ sites and for each sequenced read. Each row represents an individual sequencing read, whilst each column reflects a different $\mathrm{CpG}$ within the sequence as labelled. Methylated $\mathrm{CpG}$ sites are labelled red; unmethylated sites in blue; and unaligned in white. (c) Pearl necklace plots, again visualising example methylation data per $\mathrm{CpG}$ site. However, rather than separated reads, these plots illustrate the proportionate methylation level at each $\mathrm{CpG}$, across all sequenced reads for that sample, with the number of unmethylated, methylated and 'Not present' occurrences shown. Note that the pearl necklace plot shown in (c) does not correspond directly to the data shown in (b). This is because the heatmap shown in (c) only shows a portion of the total data set to provide an example of a methylation heatmap and (c) only shows an example of the first $22 \mathrm{CpG}$ sites. 
dCas9-SunTag

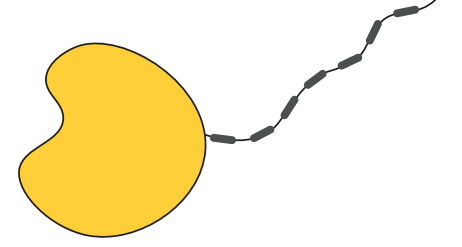

sgRNA

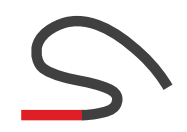

scFv-Effector

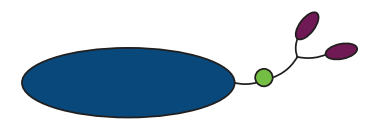

SV40 Promoter

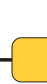

$d$ Cas 9

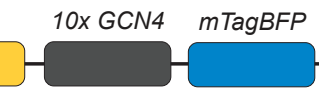

$14.5 \mathrm{~kb}$
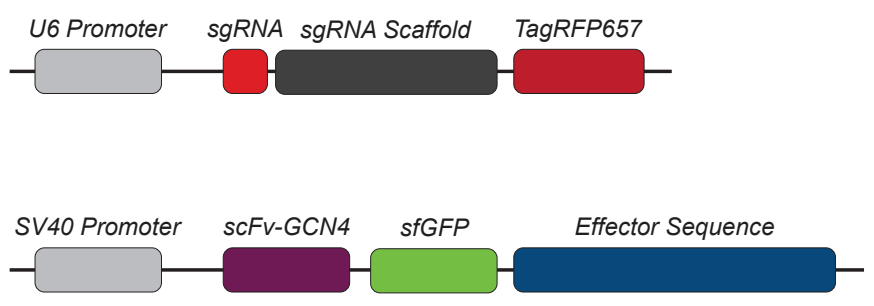

(c)

Restriction Cloning, Plasmid Propagation and DNA Isolation
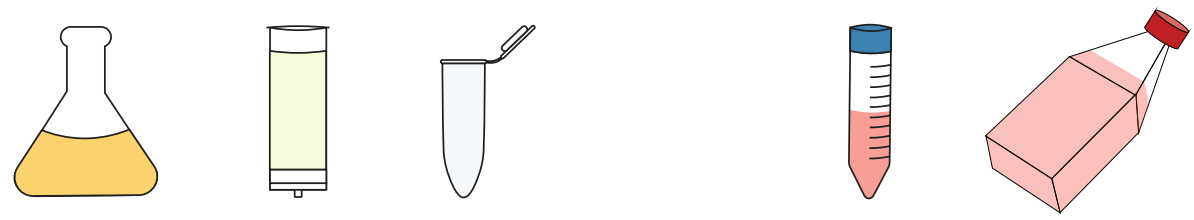

Cell Culture and Transfection Preparation
System Delivery and Targeted DNA Methylation Editing

Fluorescence-Activated Cell Sorting

Targeted DNA Methylation Sequencing and Donstream Analyses
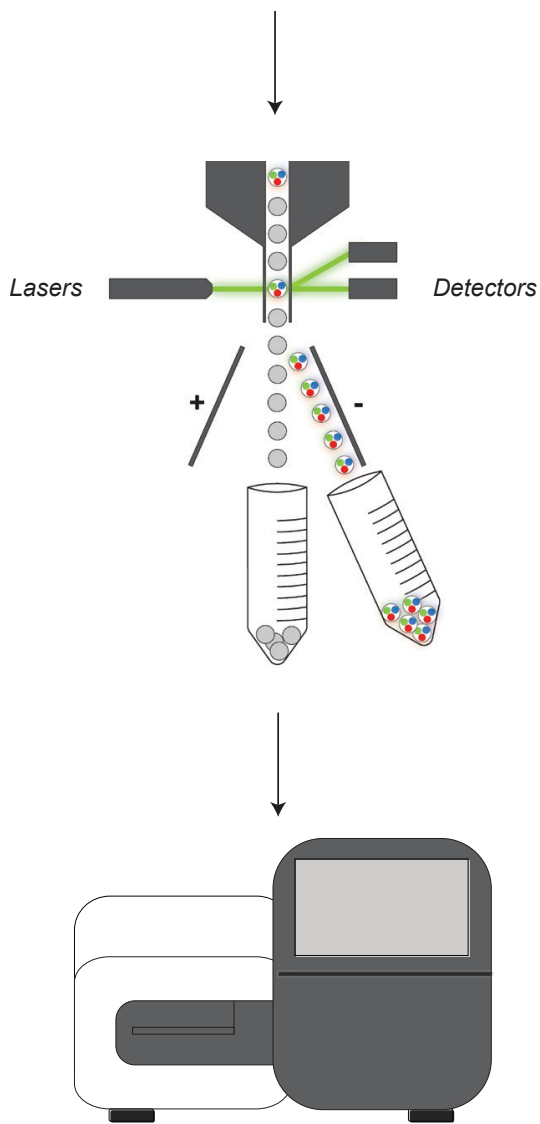

Methylated Cytosine

Unmethylated Cytosine

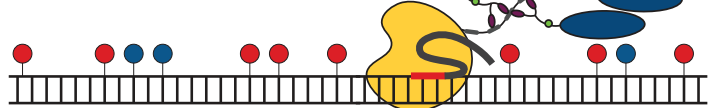

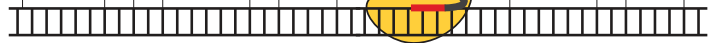




\section{EBF3 TSS}

\section{Promoter Target Region}
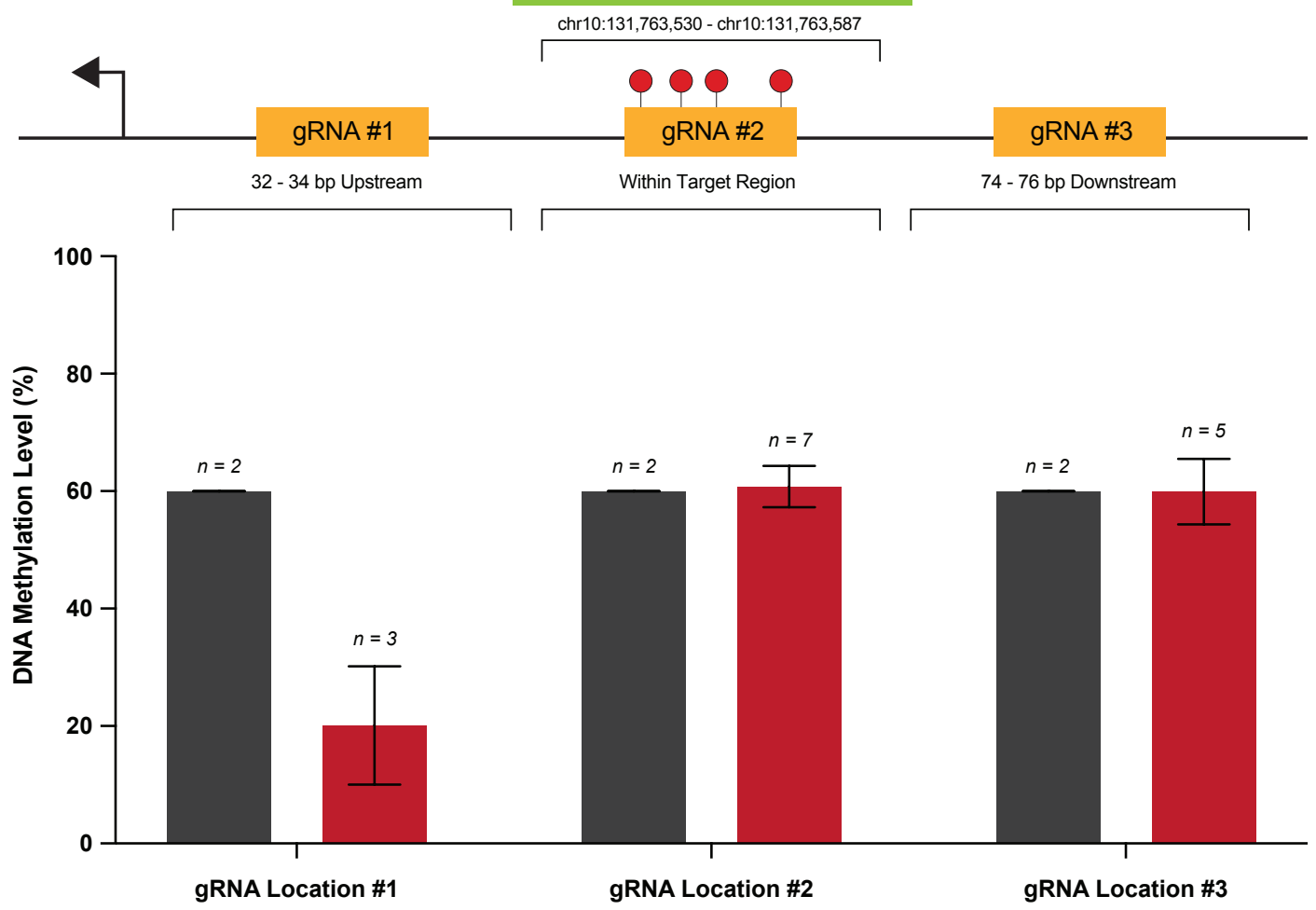

Unedited Edited 

preprint (which was not certified by peer review) is the author/funder, who has granted bioRxiv a license to display the pr
EBF3p\$\$Stuity. It is made available under aCC-BY-ND 4.0 International license.

(a)

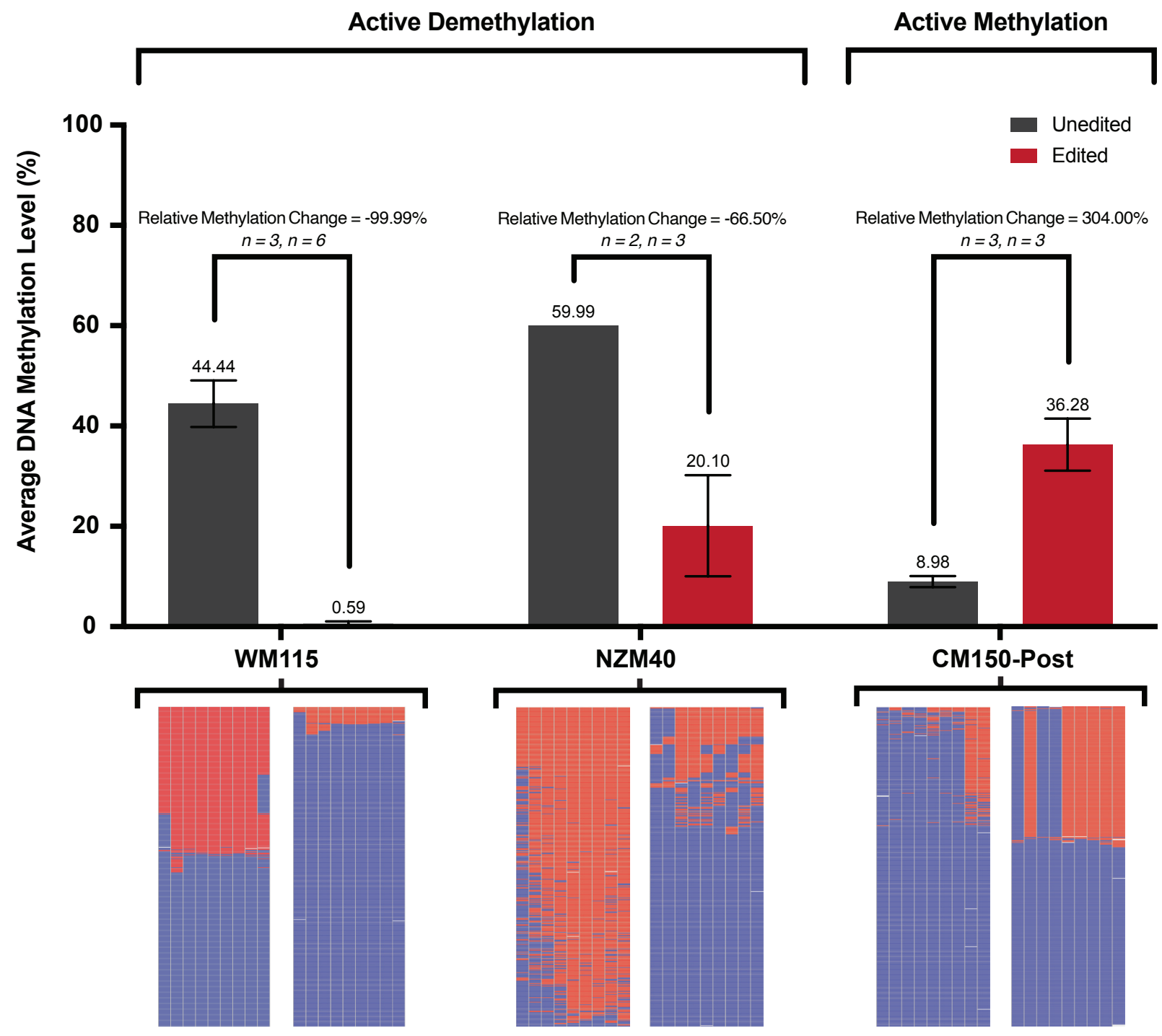

(b)

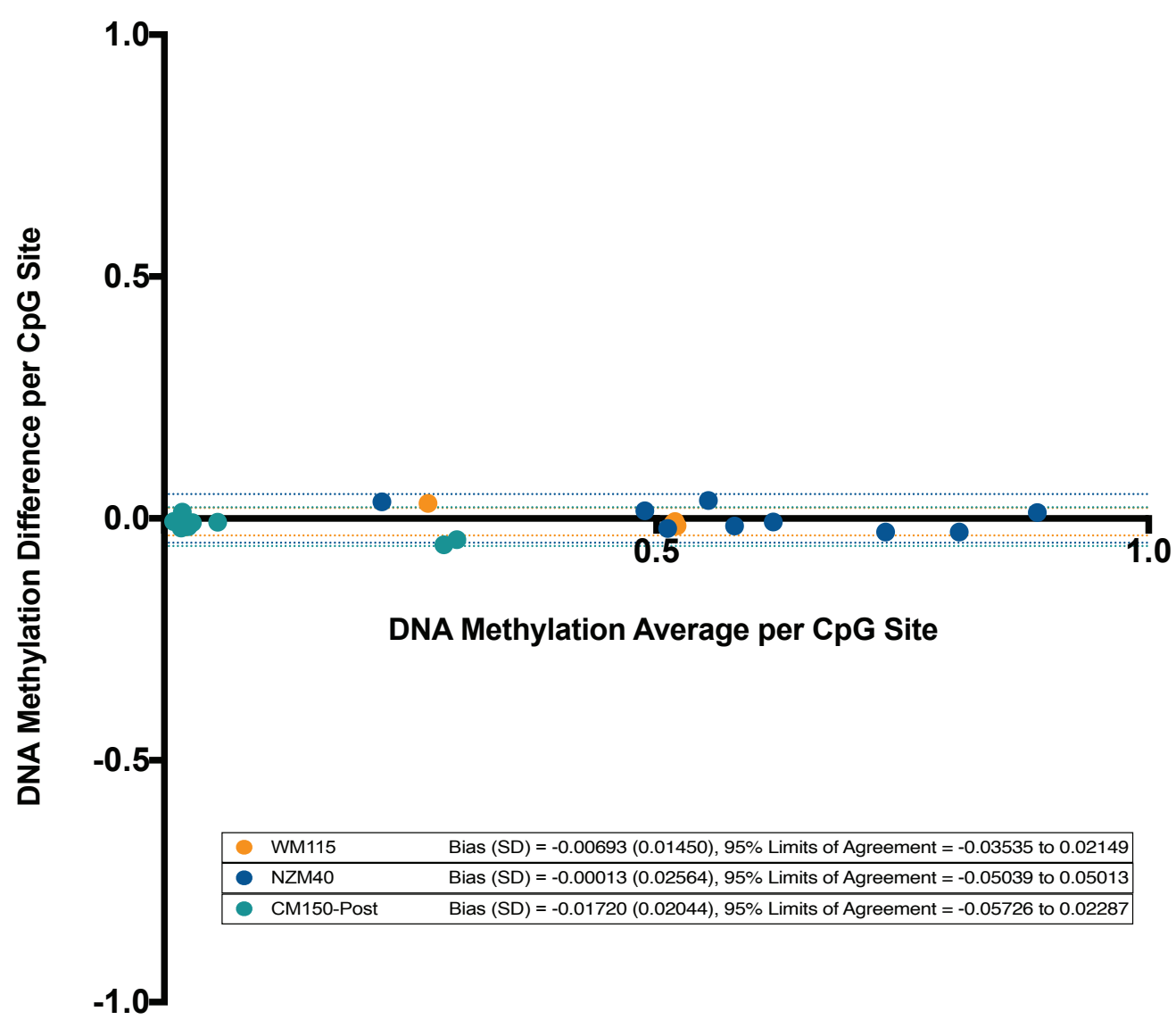




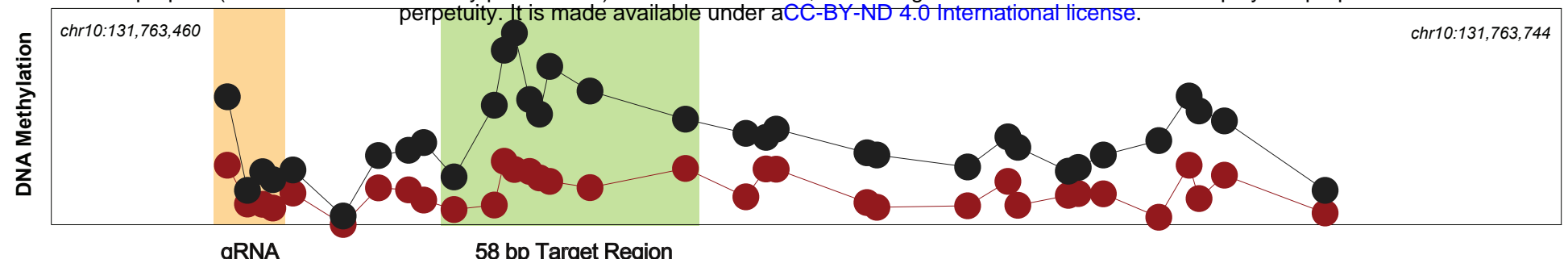

Absolute DNA Methylation Change per CpG

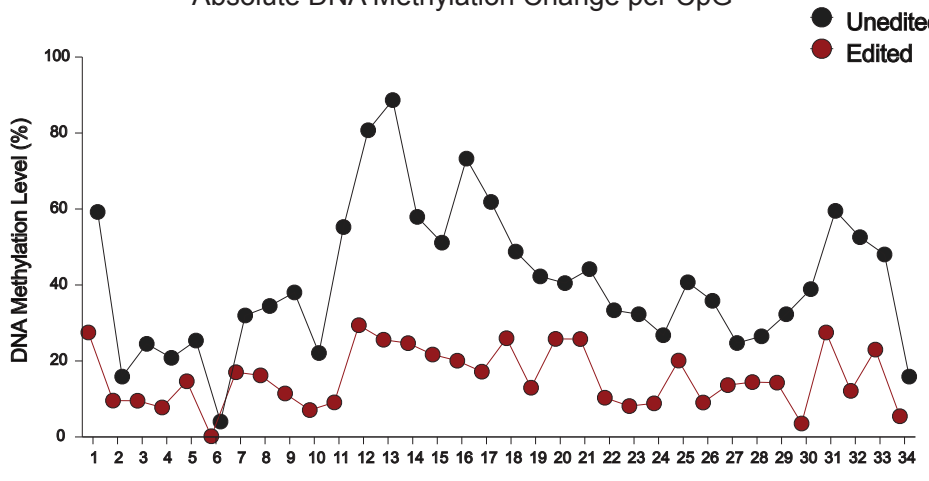

CpG Site

Relative DNA Demethylation

\% Change

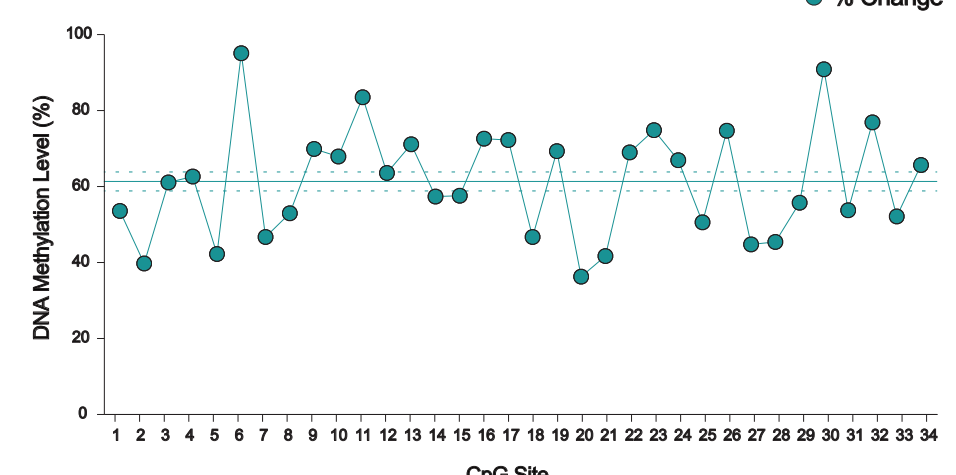
Cpg Site

WM115

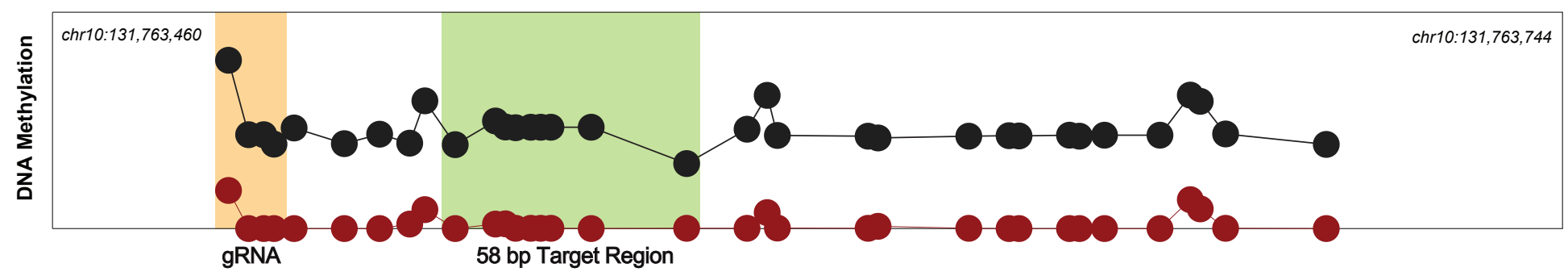

Absolute DNA Methylation Change per CpG

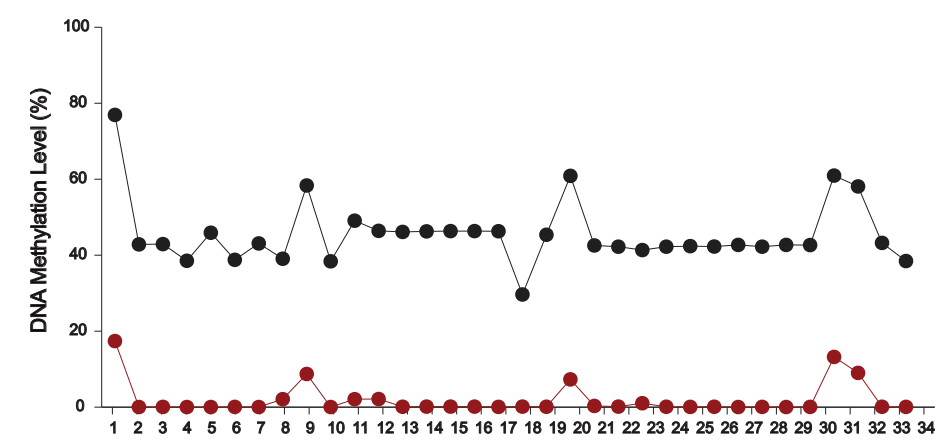

Relative DNA Demethylation

(b) CpG Site
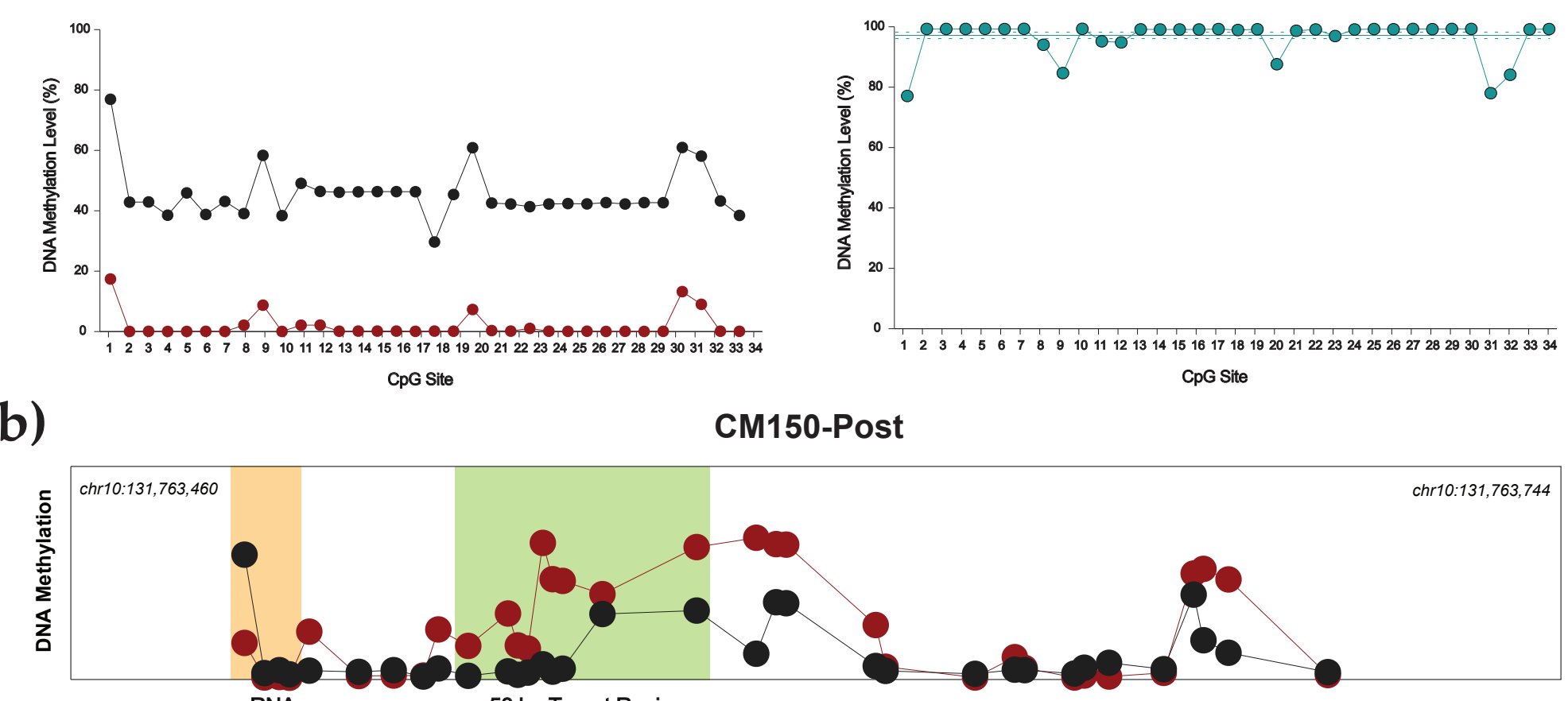

gRNA

58 bp Target Region

Absolute DNA Methylation Change per CpG

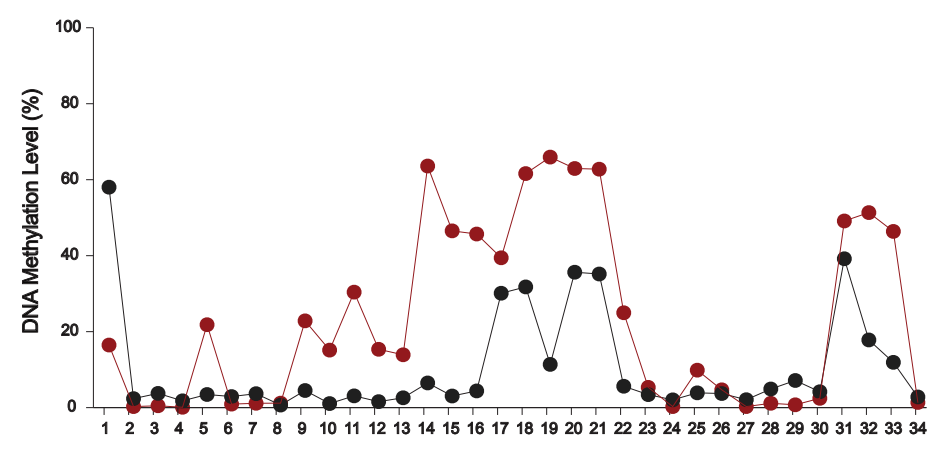

CpG Site
Relative DNA Methylation

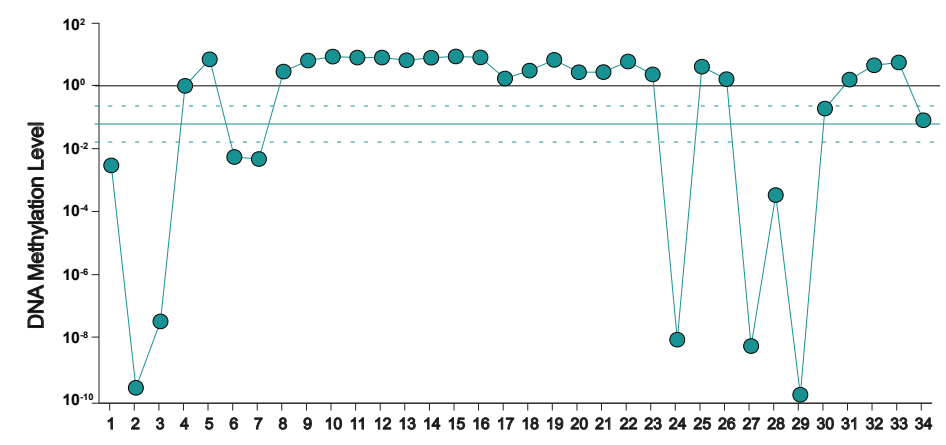


(a)

Targeted Cas 9 Cleavage

NHEJ-Mediated INDEL Formation

Sequencing of Predicted Onand Off-Targets

Cas9 + gRNA

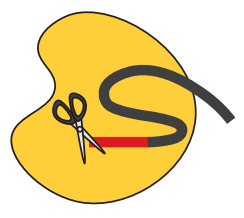

do

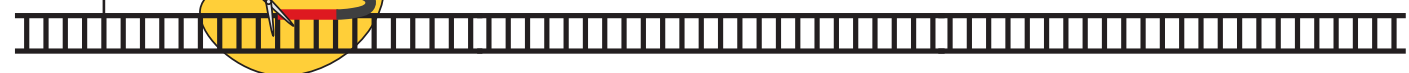
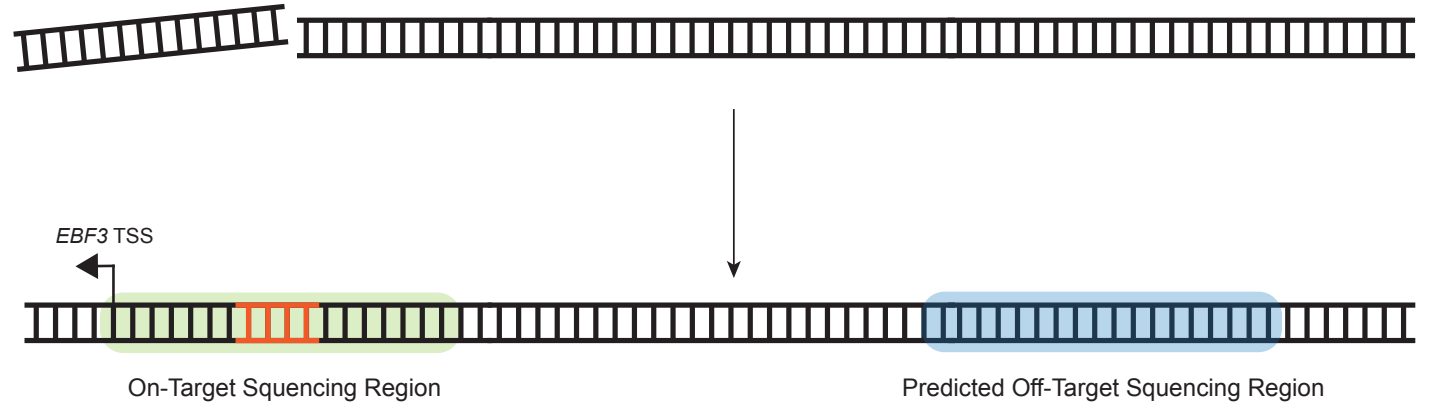

Predicted Off-Target Squencing Region

(b)

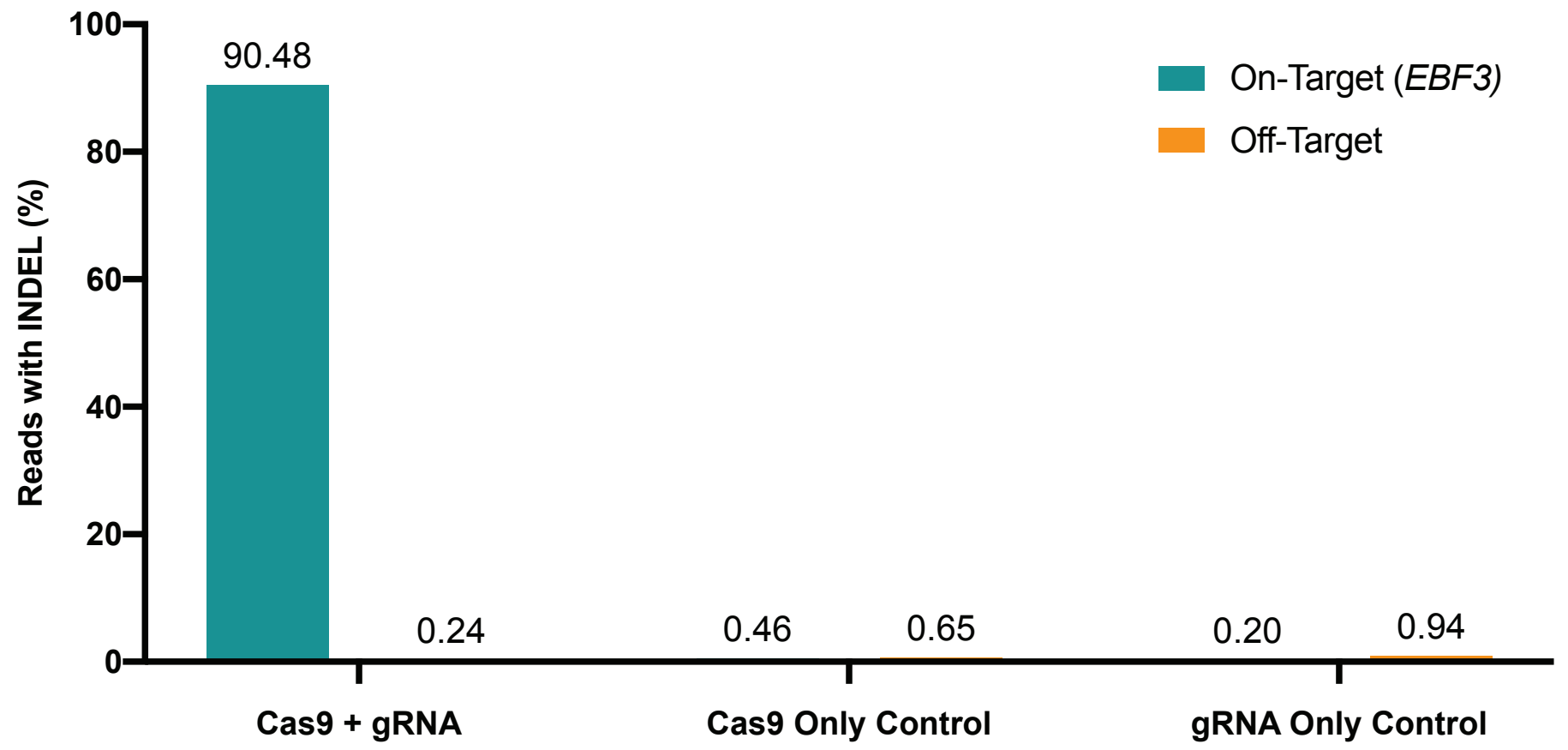



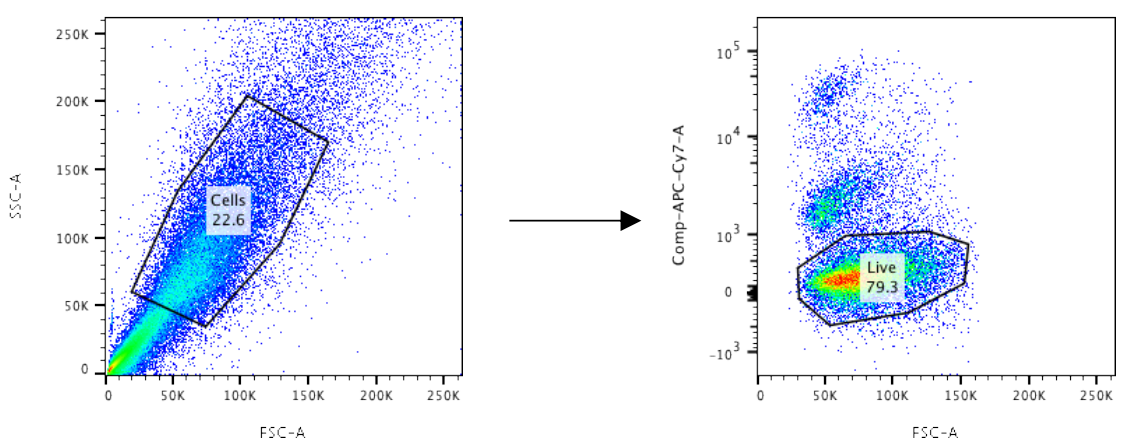

\section{Gating for Fluorophore-Positive Cells}
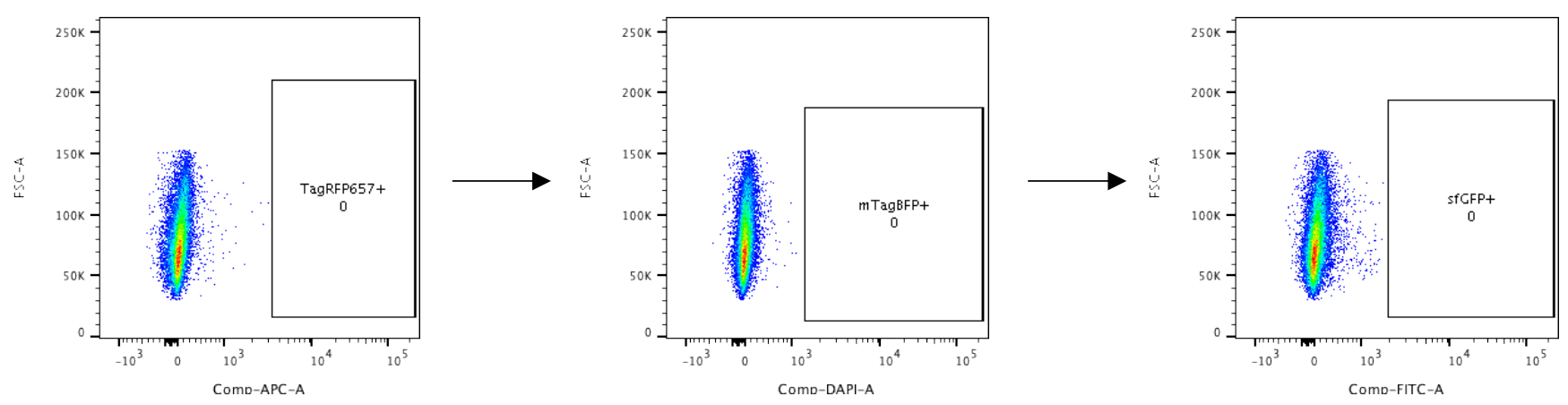

(b)
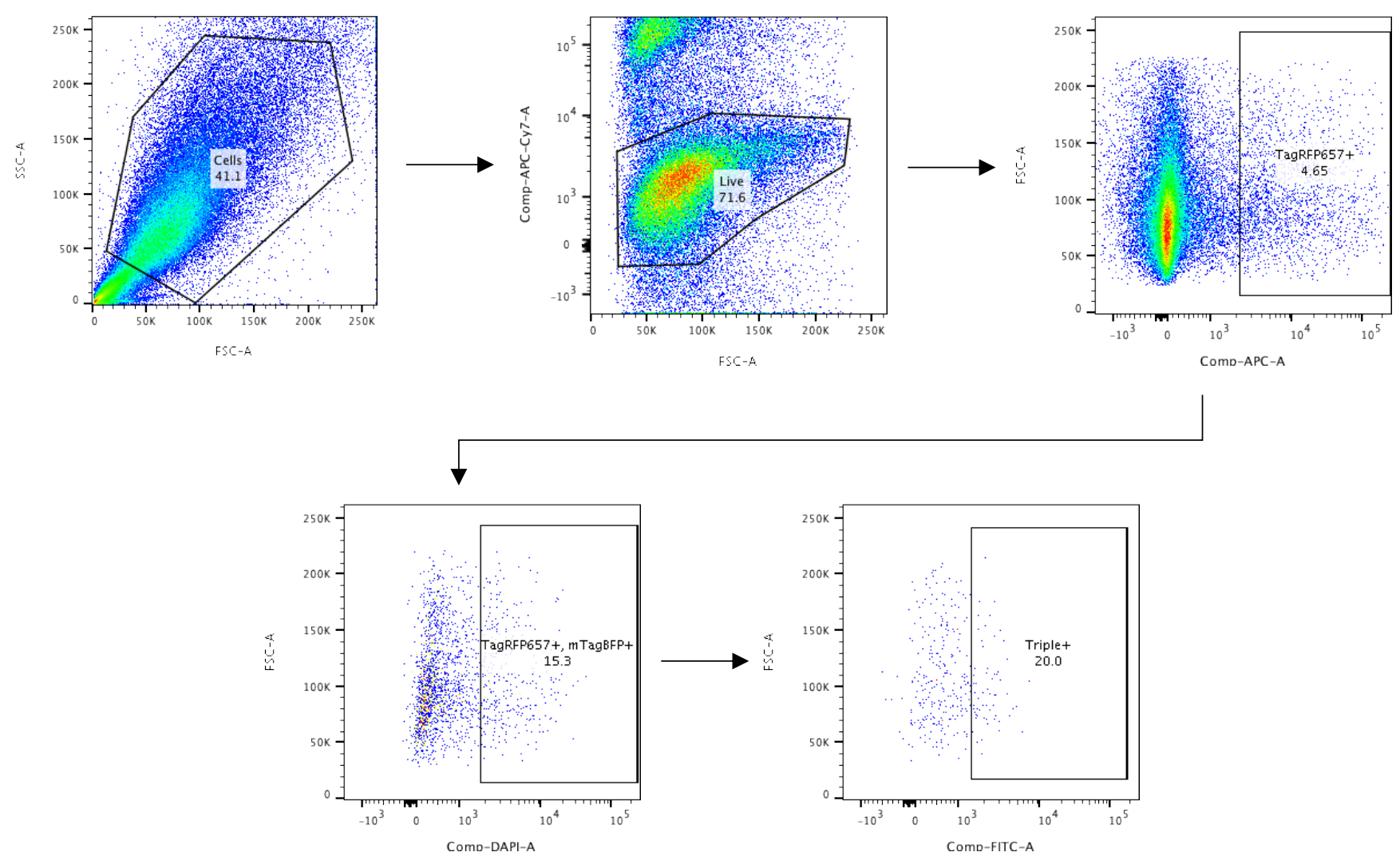
(a)

\begin{tabular}{|c|c|c|c|c|c|c|c|c|}
\hline$\Delta$ & A & B & c & D & E & $\mathrm{F}$ & G & H \\
\hline 1 & ID & Alignment_Score & Sequence_Identity & CG_Methylation_Pattern & Mean_CG_Methylation & Missing_CG_Number & Conversion & Reference \\
\hline 2 & M00733:159:000000000-D4C7F:1:1101:1839:15264 & 156 & 0.157 & 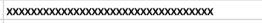 & $\mathrm{NaN}$ & 34 & 0.2353 & EBF3_genome \\
\hline 3 & M00733:159:000000000-D4C7F:1:1101:18542:25532 & 156 & 0.157 & $x x x x x x x x x x x x x x x x x x x x x x x x x x x x x x x x x x x$ & $\mathrm{NaN}$ & 34 & 0.2353 & EBF3_genome \\
\hline 4 & M00733:159:000000000-D4C7F:1:1102:9473:5584 & 156 & 0.157 & 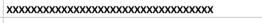 & $\mathrm{NaN}$ & 34 & 0.2353 & EBF3_genome \\
\hline 5 & M00733:159:000000000-D4C7F:1:1102:11627:17258 & 146 & 0.1535 & $x x x x x x x x x x x x x x x x x x x x x x x x x x x x x x x x x x x$ & $\mathrm{NaN}$ & 34 & 0.2353 & EBF3_genome \\
\hline 6 & M00733:159:000000000-D4C7F:1:1101:21068:4475 & 1618 & 0.9969 & 1001111111111111111110111111111111 & 0.9118 & 0 & 0.5 & EBF3_genome \\
\hline 7 & M00733:159:000000000-D4C7F:1:1101:21056:4490 & 1623 & 1 & 1001111111111111111110111111111111 & 0.9118 & 0 & 0.5147 & EBF3_genome \\
\hline 8 & M00733:159:000000000-D4C7F:1:1101:8428:5303 & 1611 & 0.9969 & 1001111111111111111110111111111111 & 0.9118 & 0 & 0.5 & EBF__genome \\
\hline 9 & M00733:159:000000000-D4C7F:1:1101:8407:5304 & 1611 & 0.9969 & 1001111111111111111110111111111111 & 0.9118 & 0 & 0.5 & EBF3_genome \\
\hline 10 & M00733:159:000000000-D4C7F:1:1101:8419:5320 & 1611 & 0.9969 & 1001111111111111111110111111111111 & 0.9118 & 0 & 0.5 & EBF3_genome \\
\hline 11 & M00733:159:000000000-D4C7F:1:1101:22695:9228 & 1623 & 1 & 1001111111111111111110111111111111 & 0.9118 & 0 & 0.5147 & EBF3_genome \\
\hline 12 & M00733:159:000000000-D4C7F:1:1101:22682:9243 & 1623 & 1 & 1001111111111111111110111111111111 & 0.9118 & 0 & 0.5147 & EBF3_genome \\
\hline 13 & M00733:159:000000000-D4C7F:1:1101:26224:10113 & 1623 & 1 & 1001111111111111111110111111111111 & 0.9118 & 0 & 0.5147 & EBF3_genome \\
\hline 14 & M00733:159:000000000-D4C7F:1:1101:23850:10883 & 1623 & 1 & 1001111111111111111110111111111111 & 0.9118 & 0 & 0.5147 & EBF3_genome \\
\hline 15 & M00733:159:000000000-D4C7F:1:1101:10554:19761 & 1623 & 1 & 1001111111111111111110111111111111 & 0.9118 & 0 & 0.5147 & EBF3_genome \\
\hline 16 & M00733:159:000000000-D4C7F:1:1101:17307:20935 & 1623 & 1 & 1001111111111111111110111111111111 & 0.9118 & 0 & 0.5147 & EBF3_genome \\
\hline 17 & M00733:159:000000000-D4C7F:1:1101:27237:23169 & 1611 & 0.997 & 1001111111111111111110111111111111 & 0.9118 & 0 & 0.5147 & EBF3_genome \\
\hline 18 & M00733:159:000000000-D4C7F:1:1101:27254:23174 & 1611 & 0.997 & 1001111111111111111110111111111111 & 0.9118 & 0 & 0.5147 & EBF3_genome \\
\hline 19 & M00733:159:000000000-D4C7F:1:1101:22131:23229 & 1623 & 1 & 1001111111111111111110111111111111 & 0.9118 & 0 & 0.5 & EBF3_genome \\
\hline 20 & M00733:159:000000000-D4C7F:1:1101:22160:23247 & 1618 & 0.9969 & 1001111111111111111110111111111111 & 0.9118 & 0 & 0.4853 & EBF__genome \\
\hline
\end{tabular}

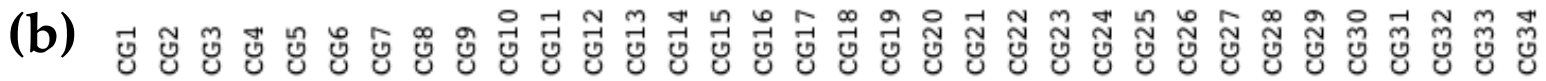

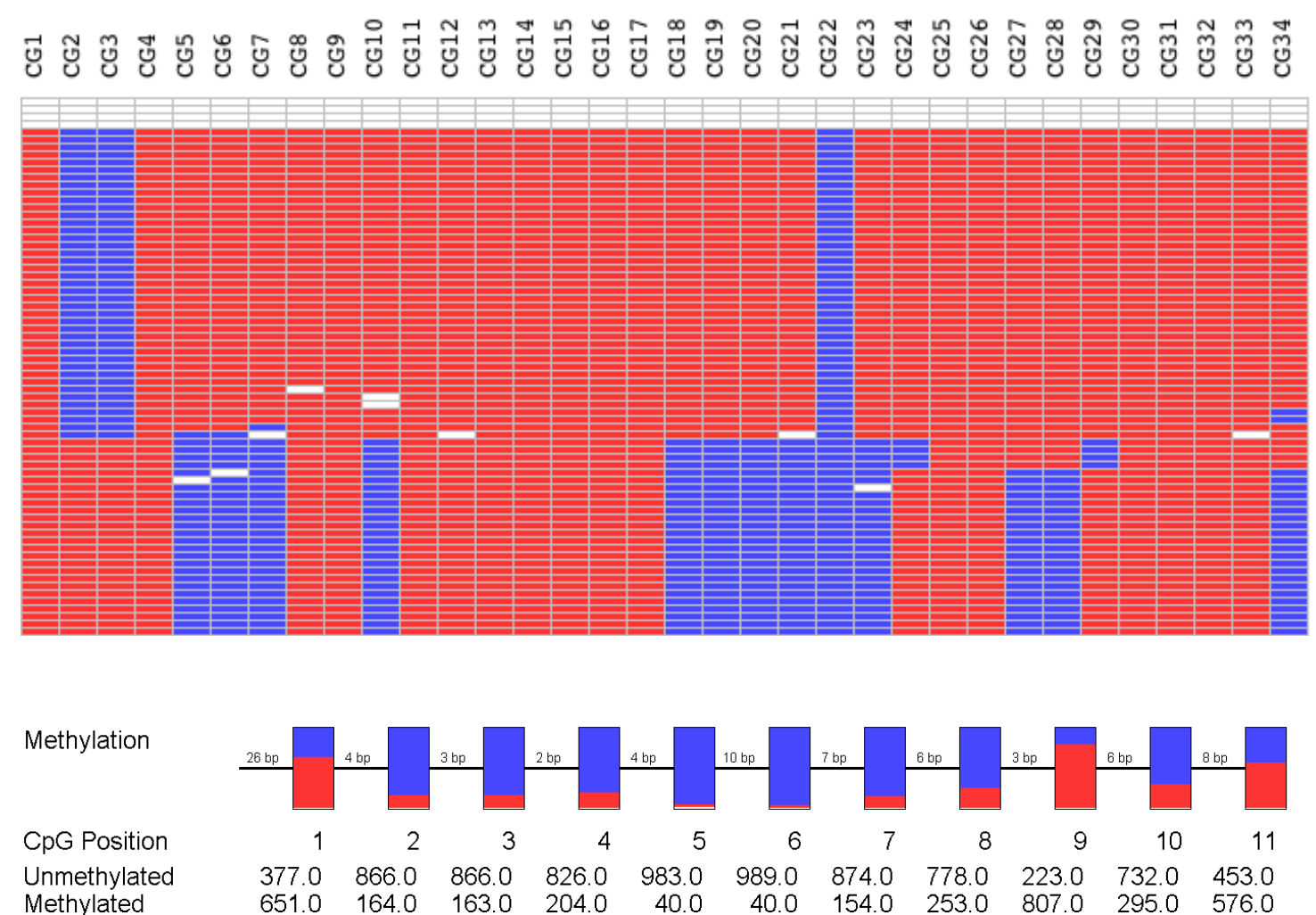

(c) Methylation

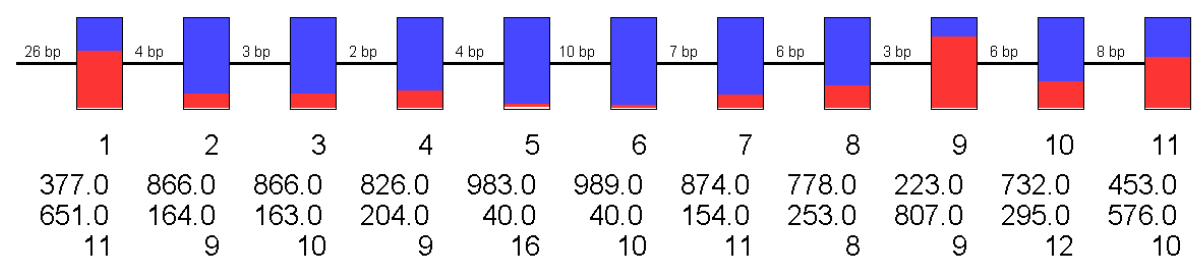

Methylation

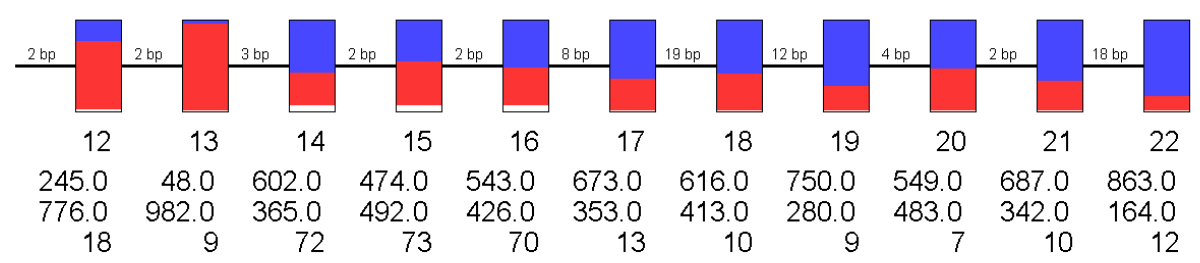

Cpg Position

Unmethylated

Methylated

Not present

CpG Position

Unmethylated

Methylated

Not present

11 